\title{
Die Konjunktiv-II-Bildung im Kontext von Partikelverben in den Basisdialekten Salzburgs
}

\author{
Sonja Quehenberger (Wien), Lars Bülow (Wien) und Philip C. Vergeiner (Salzburg)
}

\begin{abstract}
Focusing on phrasal verbs such as einbringen 'to harvest' or abbringen 'to dissuade', the aim of the present exploratory apparent-time study is to uncover factors affecting the subjunctive II formation in the traditional base dialects of Salzburg (Austria). Depending on whether the subjunctive II formation is synthetic or periphrastic, phrasal verbs are formed in contact position (e. g., würde/täte abbringen 'would dissuade') or in distance position (e. g. brächte ab 'would dissuade') between particle and verb stem.

In order to examine which subjunctive II variants are used for 15 phrasal verbs in the traditional dialects of Salzburg, an indirect survey was carried out with 25 informants in six rural locations. These villages are spread across the three dialect areas (West-Central Bavarian, South-Central Bavarian, and South Bavarian) cutting through the federal state of Salzburg. Furthermore, the subjunctive II formation of the phrasal verbs is compared to that of the simple verbs (e. g., bringen 'to bring') corresponding to the derivational bases of the respective phrasal verbs investigated (e. g., einbringen or abbringen). In addition to linguistic and areal factors, sociolinguistic factors (age and gender) are taken into account.

Results show that the subjunctive II formation of phrasal verbs differs significantly from that of simple verbs: compared to the corresponding simple verbs, the informants used the phrasal verbs significantly more often with periphrastic variants. It is argued that this tendency is related to specific semantic-lexical and morpho-syntactic properties of phrasal verbs. Regarding the areal distribution, there are differences between the individual locations. These differences do not correlate with the traditional dialect regions but can be traced back to the socio-demographic characteristics of the locations (number of inhabitants, transport connections, tourism etc.). Moreover, age proves to be a relevant factor: there is a statistically significant difference in both, simple verbs and phrasal verbs, with the younger informants preferring the periphrastic variants.
\end{abstract}

\section{$1 \quad$ Problemaufriss}

Obwohl die Konjunktiv-II-Bildung in den bairischen Dialekten Österreichs bereits mehrfach aus variationslinguistischer Perspektive untersucht wurde (cf. Breuer/Wittibschlager 2020; Stöckle 2020; Vergeiner/Bülow 2022), ist über den Einfluss bestimmter innersprachlicher Faktoren immer noch relativ wenig bekannt. 
Im Fokus der bisherigen Studien steht zumeist die Frage, ob die Konjunktiv-II-Bildung in den bairischen Dialekten Österreichs allgemein mit der Verbklasse (cf. Breuer/Wittibschlager 2020: 154; Stöckle 2020: 157f.) oder mit spezifischen Verblexemen (cf. Vergeiner/Bülow 2022) zusammenhängt. Nur wenige Erkenntnisse gibt es allerdings bislang über den Einfluss (wortbildungs-)morphologischer und syntaktischer Faktoren. Diese wurden bisher entweder nur am Rande in den Blick genommen oder ganz vernachlässigt.

Die vorliegende Studie widmet sich diesem Desiderat, wobei der Schwerpunkt auf die Konjunktiv-II-Bildung von Partikelverben gelegt wird. Diese ist deshalb besonders spannend, weil hier morpho-syntaktische und semantisch-lexikalische Eigenschaften der Verben zusammenspielen. Je nachdem, ob die Konjunktiv-II-Bildung synthetisch (z. B., zöge) oder periphrastisch (z. B., würde/täte ziehen) erfolgt, werden die Partikelverben zusammenhängend (z. B., würde/täte einziehen) oder getrennt (z. B., zöge ein) realisiert. Die periphrastische KonjunktivII-Bildung ermöglicht somit eine morphologische Kontaktstellung zwischen Verbpartikel und Basisverb (Bsp. (1)-(3)). Bei der synthetischen Konjunktiv-II-Bildung hingegen stehen Verbpartikel und Basisverb im Verberst- (4) und Verbzweitsatz (5) - jedoch nicht im Verbletztsatz (6) - in Distanzstellung.

(1) Würde/Täte Peter doch bloß bei mir einziehen!

(2) Peter würde/täte gerne bei mir einziehen.

(3) Wenn Peter doch bloß bei mir einziehen würde/täte!

(4) Zöge Peter doch bloß bei mir ein!

(5) Peter zöge gerne bei mir ein.

(6) Wenn Peter doch bloß bei mir einzöge!

Die synthetische Konjunktiv-II-Bildung bei Partikelverben weist demzufolge die Besonderheit gegenüber einfachen Verben auf, dass mithilfe dieser Konstruktionen eine Satzklammer gebildet wird (Bsp. (4) und (5)) (siehe auch Tabelle 1).

\begin{tabular}{|l|l|l|l|}
\hline \multicolumn{2}{|l|}{ einfache Verben } & Partikelverben \\
\hline synthetisch & periphrastisch & synthetisch & periphrastisch \\
\hline zöge & würde/täte ziehen & zöge ein & würde/täte einziehen \\
\hline
\end{tabular}

Tabelle 1: Synthetische und periphrastische Konjunktiv-II-Bildung von einfachen Verben und Partikelverben

Für diesen Beitrag ist entscheidend, dass die Distanzstellung von Verbpartikel und Basisverb eine Abweichung von dem von Behaghel (1932: 4) formulierten Präferenzgesetz der Nähe darstellt: „Das oberste Gesetz ist dieses, daß das geistig eng Zusammengehörige auch eng zusammengestellt wird“. Unter kognitiven Gesichtspunkten gewährleiste dies eine einfachere Prozessierung sprachlicher Strukturen. Insofern Partikelverben eine lexikalisch-semantische und wortbildungsmorphologische Einheit bilden, ist demzufolge eine Präferenz für Konjunktiv-IIBildungen mit Kontaktstellung zwischen Basisverb und Verbpartikel zu erwarten.

Um dem Status der verschiedenen Klammerbildungen gerecht zu werden, ist es allerdings nicht nur wichtig, zwischen der periphrastischen Konjunktiv-II-Klammer (Konjugation des Verbs) und der Klammerbildung beim Partikelverb (Wortbildung, Distanzstellung) zu unterscheiden, sondern auch, die semantisch-lexikalischen Eigenschaften der Partikelverben zu berück- 
sichtigen. So stellt sich bei Partikelverben die Frage, ob durch die Linkserweiterung auch tatsächlich die Bedeutung modifiziert wird (z. B., ziehen - einziehen) oder nicht (z. B., borgen ausborgen). In solchen Fällen, in denen die Linkserweiterung mithilfe der Partikel keinen Bedeutungsunterschied hervorruft, ist die Gefahr einer fehlerhaften (i. e. zu revidierenden) syntaktischen und semantischen Prozessierung geringer. Das hat zur Folge, dass die Unterscheidung zwischen Kontakt- und Distanzstellung hier weniger relevant sein dürfte als bei solchen Verben, bei denen es einen Bedeutungsunterschied gibt (siehe Kapitel 2.2).

Mit dem Fokus auf die Konjunktiv-II-Bildung in den Basisdialekten Salzburgs wird im vorliegenden Beitrag ein Untersuchungsgebiet in den Blick genommen, das sich trotz all seiner sprachgeografischen Unterschiede (siehe Abbildung 1) grundsätzlich durch eine starke Verankerung der synthetischen Bildungsstrategie bei einfachen Verben auszeichnet (cf. Vergeiner/Bülow 2022; Stöckle 2020: 157-161; Mauser 1998: 342-355). Das hat den Vorteil, dass sich relativ einfach mögliche Unterschiede zur Konjunktiv-II-Bildung bei Partikelverben aufzeigen lassen sollten.

Um zu verstehen, wie sich die semantisch-lexikalischen und morpho-syntaktischen Eigenheiten von Partikelverben auf die Konjunktiv-II-Bildung in den Basisdialekten Salzburgs auswirken, hat diese explorative Studie zum Ziel, die folgenden Forschungsfragen (FF) zu klären:

FF1: Welche Varianten der Konjunktiv-II-Bildung kommen bei Partikelverben in den Basisdialekten Salzburgs zum Einsatz? Unterscheidet sich die Konjunktiv-II-Bildung bei Partikelverben von derjenigen bei einfachen Verben?

FF2: Erweisen sich semantisch-lexikalische Eigenschaften von Partikelverben als relevante Faktoren für die Konjunktiv-II-Bildung in den Basisdialekten Salzburgs?

FF3: Erweist sich die sprachgeografische Gliederung Salzburgs als relevanter Faktor für die Konjunktiv-II-Bildung bei Partikelverben?

FF4: Zeigen sich Wandeltendenzen bei der Konjunktiv-II-Bildung von Partikelverben in den Basisdialekten Salzburgs?

Zur Beantwortung dieser Fragen wurde eine apparent-time-Studie mithilfe einer indirekten Dialektbefragung durchgeführt. Befragt wurden 25 Gewährspersonen an sechs Orten im Bundesland Salzburg, wobei alle fünf Bezirke mit mindestens einem Untersuchungsort berücksichtigt sind. Insgesamt wurde die Konjunktiv-II-Bildung bei 15 Partikelverben mit den Linkserweiterungen $a n-, a b-$, auf- und ein- untersucht. Neben den Partikelverben wurden auch die den Basisverben der Partikelverbkonstruktionen entsprechenden einfachen Verben analysiert, um mögliche Unterschiede herauszuarbeiten. Um eine möglichst hohe Vergleichbarkeit zu gewährleisten, wurden die einfachen Verben der Studie von Vergeiner/Bülow (2022) entnommen.

Der Beitrag gliedert sich wie folgt: Nachfolgend wird der Forschungsstand (Kapitel 2) zum Konjunktiv II im Bairischen (Kapitel 2.1) und zu Partikelverben (Kapitel 2.2) dargelegt, woraufhin die Daten und Methoden (Kapitel 3) vorgestellt werden. Der Schwerpunkt des Beitrags liegt auf der Präsentation der Ergebnisse (Kapitel 4) und ihrer Diskussion (Kapitel 5). Den Abschluss bildet ein kurzes Fazit (Kapitel 6). 


\section{Forschungsstand}

Im Folgenden wird zunächst der Forschungsstand zum Konjunktiv II in den bairischen Dialekten dargestellt (Kapitel 2.1), bevor die morpho-syntaktischen und semantisch-lexikalischen Eigenschaften von Partikelverben genauer erörtert werden (Kapitel 2.2).

\subsection{Der Konjunktiv II im Bairischen}

Im Zuge der verstärkten Bemühungen zur Erforschung der Dialektsyntax in den vergangenen Jahrzehnten (siehe zum Beispiel SADS; SyHD) sind auch Variation und Wandel der Konjunktiv-II-Bildung in den bairischen Dialekten Österreichs in den Fokus variationslinguistischer Forschungsarbeiten gerückt. Neben Arbeiten von Glauninger (2008, 2010, 2011) und Lenzhofer (2017), die sich hauptsächlich mit sozio-pragmatischen Aspekten befassen, sind insbesondere die aktuellen Studien von Stöckle (2020), Breuer/Wittibschlager (2020) und Vergeiner/Bülow (2022) zu nennen, die die innersprachlichen, soziolinguistischen und sprachgeografischen Faktoren des Konjunktiv-II-Gebrauchs in den bairischen Dialekten Österreichs in den Blick nehmen. Stöckle (2020) wertet Daten des „Wörterbuchs der bairischen Mundarten in Österreich (WBÖ)“ aus, die aus der ersten Hälfte des 20. Jahrhunderts stammen. Die Arbeiten von Breuer/Wittibschlager (2020) und Vergeiner/Bülow (2022) stützen sich hingegen auf rezente Daten, die größtenteils zwischen 2016 und 2018 im Kontext des SFB-Projekts „Deutsch in Österreich“ erhoben wurden. Dabei basiert die Studie von Breuer/Wittibschlager (2020) auf Daten aus 11 ruralen Ortspunkten und Wien, die mithilfe sogenannter Sprachproduktionsexperimente erhoben wurden. Vergeiner/Bülow (2022) analysieren Daten aus einer flächendeckenden direkten Dialektbefragung an 40 Orten mit 163 Gewährspersonen. Bevor genauer auf die Ergebnisse dieser Studien eingegangen wird, ist zunächst das Formeninventar des Konjunktiv II in den bairischen Dialekten zu erläutern.

\subsubsection{Formen}

Der Konjunktiv II kann im Bairischen mithilfe zweier Strategien gebildet werden: synthetisch, i. e. morphologisch mittels Stammmodulation und/oder Flexionssuffixen, und periphrastisch, i. e. syntaktisch unter Verwendung von Hilfsverben.

Synthetische Formen werden schwach, stark oder gemischt gebildet. Schwache Verben bilden den Konjunktiv II, indem sie am Präsensstamm das Suffix -at annehmen (i kafat, ich kaufte'). Starke Verben können durch Stammvokalwechsel und Nullendung flektiert werden (i las ,ich läse'), sie können jedoch auch mithilfe der -at-Markierung am Präsens- oder Präteritumstamm schwache $(i$ lesad) oder gemischte Konjunktivformen (i lasad) ausbilden (cf. Stöckle 2020: 151f.). Da Partikelverben grundsätzlich die Flexionsklasse ihres Basisverbs übernehmen (cf. Stiebels 1996: 38), unterscheiden sie sich hinsichtlich ihrer Flektierbarkeit mit oder ohne -at nicht von den zugrundeliegenden Simplizia. Bei der synthetischen Konjunktiv-II-Bildung stehen Partikel und Basisverb im Verberst- und Verbzweitsatz jedoch in Distanzstellung (siehe Kapitel 1).

Periphrastische Varianten werden entweder mit tun- oder würde-Auxiliar gebildet (z. B. i dat lesn). Daneben existieren periphrastische Hybridformen mit tun-oder würde-Auxiliar und -atSuffix (z. B. i darat lesn). Im Falle der periphrastischen Konjunktiv-II-Bildung stehen Verbpartikel und Basisverb in morphologischer Kontaktstellung. 
Tabelle 2 fasst die synthetischen und periphrastischen Varianten der Konjunktiv-II-Bildung für einfache Verben und Partikelverben zusammen:

\begin{tabular}{|l|l|l|l|l|l|}
\hline \multicolumn{5}{|c|}{ synthetisch } \\
\hline Echwach & gemischt & stark & schwach & gemischt & stark \\
\hline les-at & las-at & las & les-at voa & las-at voa & las voa \\
\hline \multicolumn{5}{|c|}{ periphrastisch } \\
\hline \multicolumn{3}{|c|}{ Einfache Verben } & \multicolumn{4}{|c|}{ Partikelverben } \\
\hline tun-Periphrasen & würde-Periphrasen & tun-Periphrasen & würde-Periphrasen \\
\hline $\begin{array}{l}\text { dat lesn } \\
\text { dar-at lesn }\end{array}$ & $\begin{array}{l}\text { wuat lesn } \\
\text { wuad-at lesn }\end{array}$ & $\begin{array}{l}\text { dat voalesn } \\
\text { dar-at voalesn }\end{array}$ & $\begin{array}{l}\text { wuat voalesn } \\
\text { wuad-at voalesn }\end{array}$ \\
\hline
\end{tabular}

Tabelle 2: Synthetische und periphrastische Varianten der Konjunktiv-II-Bildung für einfache Verben und Partikelverben im Bairischen ${ }^{1}$

\subsubsection{Steuerungsfaktoren}

Wie oben bereits angedeutet wurde, zeigen aktuelle Forschungsarbeiten zur Konjunktiv-II-Bildung in den bairischen Dialekten Österreichs, dass die Wahl spezifischer Varianten sowohl von innersprachlichen als auch von sprachgeografischen und soziolinguistischen Faktoren gesteuert wird (cf. Stöckle 2020; Breuer/Wittibschlager 2020; Vergeiner/Bülow 2022). Im Folgenden werden diese einzelnen Faktoren näher beleuchtet.

Als relevante innersprachliche Faktoren erweisen sich sowohl die Verbklasse (cf. Stöckle 2020; Breuer/Wittibschlager 2020) als auch verbspezifische Eigenschaften (cf. Vergeiner/Bülow 2022). Stöckle (2020: 157f.) zeigt anhand des WBÖ-Materials aus der ersten Hälfte des 20. Jahrhunderts, dass bei schwachen Verben bis auf wenige Ausnahmen die synthetische Variante mit -at-Suffix dominiert. Bei starken Verben überwiegt zwar ebenfalls die Variante mit -at-Suffix, jedoch ist in 39\% der Fälle auch die starke synthetische Form ohne -at-Suffix dokumentiert. Unregelmäßige Verben wie haben und sein bilden den Konjunktiv II mehrheitlich durch die starke synthetische Form.

Eine verbklassenspezifische Verteilung der Konjunktiv-II-Varianten zeigt sich auch in den rezenten Dialektdaten von Breuer/Wittibschlager (2020: 154). Während unregelmäßige und starke Verben bei ihnen eine ausgewogene Verteilung zwischen synthetischen und periphrastischen Varianten aufweisen, werden schwache Verben in überwiegender Mehrheit periphrastisch realisiert.

Der Einfluss der Verbklassen auf die Wahl verschiedener Konjunktiv-II-Varianten kann in der ebenfalls auf aktuellen Dialektdaten beruhenden Studie von Vergeiner/Bülow (2022) nur bedingt bestätigt werden: Die 21 von ihnen untersuchten Verben, die unterschiedlichen Verbklassen (stark, schwach, Modalverben, besondere Verben) angehören, werden mittels Clusteranalyse in sechs Cluster eingeteilt. Bemerkenswert ist, dass die Zugehörigkeit zu einer bestimmten Flexionsklasse die Konjunktiv-II-Variation nur teilweise zu beeinflussen scheint: So bündelt

\footnotetext{
${ }^{1}$ Lautliche Aspekte wurden hier vernachlässigt. Bei den synthetischen Formen wurden nur die prototypischen Flexionsparadigmen (cf. Stöckle 2020: 151; Vergeiner/Bülow 2022) angegeben.
} 
beispielsweise das größte Cluster (Cluster 1) vier starke (ziehen, helfen, nehmen, liegen) und fünf schwache Verben (sagen, glauben, kaufen, brauchen, machen). Lediglich die suppletiven Verben sein und haben, die gemeinsam in einem Cluster (Cluster 6) zusammengefasst sind, scheinen sich entsprechend ihrer Verbklassenzugehörigkeit von den übrigen Verben abzuheben. Beide Verben bilden im gesamten Untersuchungsgebiet in 99\% der Fälle den Konjunktiv II mithilfe synthetischer Varianten.

Über die rezente sprachgeografische Variation gibt insbesondere die Untersuchung von Vergeiner/Bülow (2022) Aufschluss, da ihre Daten aus 40 Ortspunkten stammen, die gleichmäßig über die verschiedenen Dialektregionen Österreichs verteilt sind. Mithilfe einer Faktorenanalyse können sie eine großräumige Ost-West-Staffelung der Variation herausarbeiten, mit stärkerer Tendenz zu synthetischen Formen, allen voran der Variante mit -at-Suffix, im Areal zwischen Innsbruck und Linz. In den alemannischen Dialektregionen sowie im Südosten des Bairischen zeigt sich hingegen bei den meisten starken und schwachen Verben eine klare Präferenz für die periphrastische Form mit tun-Auxiliar ohne -at-Suffix. Die periphrastische Variante mit tun-Auxiliar + -at-Suffix sowie die einfache würde-Periphrase sind arealunabhängig nur marginal belegt. Im hier fokussierten Bundesland Salzburg, das mit dem Westmittelbairischen, dem Südmittelbairischen und dem Südbairischen drei Dialektareale repräsentiert (cf. Wiesinger 1983), ist ausgehend von Vergeiner/Bülow (2022) kaum arealbezogene Variation, sondern vielmehr eine generelle Präferenz synthetischer Formen zu erwarten.

Hinsichtlich der soziolinguistischen Faktoren nehmen Stöckle (2020: 164-168) und Breuer/ Wittibschlager (2020: 161f.) auch die vertikale Variation (Dialekt-Standard-Variation) in den Blick. Bereits in den Daten des WBÖ-Korpus aus der ersten Hälfte des 20. Jahrhunderts zeichnet sich in standardorientierten Übersetzungen eine Tendenz $\mathrm{zu}$ würde-Periphrasen ab (cf. Stöckle 2020: 164-168). Auch die Ergebnisse von Breuer/Wittibschlager (2020: 161f.) zeigen eine stärkere Präferenz der periphrastischen Variante mit würde-Auxiliar ohne -at-Suffix in standardnahen Registern. Das kann mit dem Befund in Einklang gebracht werden, dass sich in der Untersuchung von Breuer/Wittibschlager (2020: 159f.) auch das Alter als relevanter Faktor erweist. Sowohl in Wien als auch in den rural geprägten Ortspunkten sind periphrastische Varianten signifikant häufiger bei jungen Gewährspersonen als bei älteren dokumentiert. Da die Form mit würde-Auxiliar ohne -at-Suffix bei älteren Gewährspersonen aus ländlichen Gemeinden seltener belegt ist, sprechen die Daten von Breuer/Wittibschlager (2020: 159f.) aus sprachdynamischer Perspektive für eine Zunahme der einfachen würde-Periphrase. Zwar wird der Abbau synthetischer Formen zugunsten periphrastischer Varianten durch die Studie von Vergeiner/Bülow (2022) prinzipiell bestätigt, hier zeigt sich allerdings eine Tendenz zur einfachen tun-Periphrase bei den jüngeren Gewährspersonen. Der Grund dafür dürfte der stärkere Fokus auf die Basisdialekte bei Vergeiner/Bülow (2022) sein. Neben dem Alter berücksichtigen Vergeiner/Bülow (2022) auch die Variable Geschlecht, können hierfür aber keinen signifikanten Einfluss feststellen.

\subsection{Partikelverben}

Während es einige variationslinguistisch orientierte Untersuchungen mit standardsprachlichem Fokus zu Partikelverben gibt (cf. Fingerhuth 2019; Niehaus 2015; Dürscheid/Sutter 2014), ist die entsprechende dialektologische Forschungslage, vor allem im österreichischen Raum, 
bescheiden. Eine breit angelegte Untersuchung zur Verwendung diverser Partikelverben in der Gegenwartssprache findet sich in der Variantengrammatik des Standarddeutschen (2018), jedoch liegt auch hier der Schwerpunkt auf den standardsprachlichen Varietäten. Mit Schabus (1982) existiert zwar eine dialektologische Untersuchung zu Präfix- und Partikelverben im Südbairischen, die Arbeit ist allerdings auf Kärnten beschränkt. Die im Folgenden behandelten linguistischen Faktoren, die die Konjunktiv-II-Bildung bei Partikelverben beeinflussen könnten, müssen daher primär aus Studien mit standardsprachlichem Fokus abgeleitet werden. Im Fokus dieses Beitrags steht die Frage, wie sich Partikelverben im Vergleich zu einfachen Verben auf ein synthetisches oder periphrastisches Bildungsschema auswirken: Wie bereits beschrieben wurde, erfordert die synthetische Konjunktiv-II-Bildung eine morphologische Distanzstellung von Verbpartikel und Basisverb, wohingegen die periphrastische Konjunktiv-II-Bildung eine Kontaktstellung erlaubt.

\subsubsection{Syntaktische und semantische Faktoren}

Trennbare Partikeln dienen, wie auch feste Präfixe, der syntaktischen und/oder semantischen Modifikation eines Basisverbs (cf. Fleischer/Barz 2012: 378f.; Erben 2006: 85-90). Dabei ist syntaktisch zu berücksichtigen, dass Partikelverben eine andere Argumentstruktur als ihre Basisverben aufweisen können (cf. Eisenberg 2006: 264f.): Das einfache Verb kleben hat beispielsweise einen anderen Valenzrahmen als das Partikelverb ankleben, wobei die Partikel anzu einer „Argumentsättigung“ (Eisenberg 2006: 264f.) führt, die die obligatorische Position des Präpositionalobjekts im Valenzrahmen aufhebt (Sie klebt den Zettel an die Wand vs. Sie klebt den Zettel an). Für diese explorative Studie ist entscheidend, dass bisher nicht untersucht wurde, ob sich Unterschiede im Valenzrahmen auf die Konjunktiv-II-Bildung auswirken.

Semantisch wird ein Basisverb bei Partikelverbbildungen häufig hinsichtlich der Aktionsart verändert (cf. Erben 2006: 87f.). Die hier analysierten Verbpartikeln an-, auf- und ein- signalisieren in erster Linie das Einsetzen einer Handlung (ingressive Aktionsart), die Partikel $a b$ hingegen ihren Abschluss bzw. ihre vollständige Durchführung (egressive Aktionsart) (cf. Erben 2006: 87f.; Fleischer/Barz 2012: 382, 398). Wie oben schon dargestellt wurde, ist eine solche Bedeutungsmodifikation aber nicht bei allen Partikelverben gleichermaßen der Fall, borgen und ausborgen weisen beispielsweise die gleiche Bedeutung auf. ${ }^{2}$ Bei solchen Partikelverben ist ausgehend von der fehlenden bzw. nur schwachen semantischen Modifikation des Basisverbs eine häufigere Tilgung der Verbpartikel zu erwarten. Dadurch, dass borgen und ausborgen als lexikalische Varianten zum Ausdruck derselben Bedeutung angesehen werden können, ist ferner anzunehmen, dass sich auch die Konjunktiv-II-Bildung beim einfachen Verb und Partikelverb ähnlich verhält. Bei jenen Partikelverben hingegen, bei denen die Partikel die Gesamtbedeutung stark modifiziert, dürften aus kognitiver Sicht jene synthetischen KonjunktivII-Varianten, in denen Verbpartikel und Basisverb in Distanzstellung stehen, die Prozessierung erschweren; zu untersuchen ist, ob damit eine stärkere Vermeidung synthetischer Varianten einhergeht.

\footnotetext{
${ }^{2}$ Unterschiede zeigen sich nur in der arealen Verteilung der Varianten: So stellt ausborgen in weiten Teilen Österreichs standardsprachlich die bevorzugte Variante dar, während in bundesdeutschen Standardvarietäten das einfache Verb borgen häufiger gebraucht wird (cf. Variantengrammatik des Standarddeutschen 2018 [Verben mit/ohneVerbpartikel]).
} 
Auch im Hinblick auf das Wortbildungsmuster eines Partikelverbs in standardsprachlichen Varietäten muss man, wie Erben (2006: 55), davon ausgehen, dass dieses nicht ,,in der gleichen Vollständigkeit und gleichen funktionellen Belastung auch in den deutschen Mundarten dominant" ist. So stellte etwa Schabus (1982: 21-33) fest, dass das dialektale Präfix der- mit er-, zer- und ver- gleich drei standardsprachliche Präfixe mehr oder weniger konsequent ersetzt. ${ }^{3}$ Auch Bildungen mit dem Präfix be-werden teils durch solche mit den Partikeln zu- (zudecken anstelle von bedecken) und an- (anschmieren anstelle von beschmieren) ersetzt (cf. Schabus 1982: 15, 94). Während der dialektale Präfixbestand vergleichsweise restringiert ist, ist das Partikelsystem funktional erweitert, insofern es auch Verben, die standardsprachlich bevorzugt mit festen Präfixen gebildet werden, einschließt.

\subsubsection{Lexikalisierung}

Einen weiteren potentiellen innersprachlichen Einflussfaktor stellt die Lexikalisierung von Partikelverbkonstruktionen dar. Lässt sich die Bedeutung eines Partikelverbs nicht aus seinen Einzelkomponenten, i. e. Verbpartikel und Basisverb, erschließen, so gilt es als lexikalisiert bzw. semantisch opak; im Umkehrschluss handelt es sich um transparente Partikelverbbildungen, wenn die Rekonstruktion der Gesamtbedeutung eines Partikelverbs aus seinen Einzelbestandteilen möglich ist (cf. Felfe 2012: 21-25). Die Rekonstruierbarkeit alleine reicht jedoch nicht aus: „Sprecher und Hörer müssen die Gesamtkonstruktion kennen, um die Bedeutung kompositionell aus der Konstruktion, dem Basisverb und den realisierten Argumenten der Konstruktion zu erschließen“ (Felfe 2012: 22).

So handelt es sich beim hier analysierten Partikelverb angeben (,prahlen') um ein semantisch opakes Partikelverb, da sich die Bedeutung, prahlen' nicht aus den Komponenten an- und geben erschließen lässt. Zudem lässt sich angeben (,prahlen') erst durch die syntaktische Gesamtkonstruktion und/oder den Kontext vom homonymen Verb angeben (,nennen, mitteilen') disambiguieren. Beim Partikelverb einkaufen ist das Basisverb -kaufen bedeutungstragend, die Partikel ein- signalisiert die Bewegung ,auf den eigenen Besitz“(Fleischer/Barz 2012: 411) zu. Innerhalb der Gesamtkonstruktion, in die das Partikelverb eingebettet ist, scheint zumindest standardsprachlich kein Zweifel an der lexikalischen Transparenz des Partikelverbs zu bestehen.

In vielen anderen Fällen kann jedoch auch standardsprachlich nicht eindeutig zwischen völlig opaken Bildungen einerseits und völlig transparenten Konstruktionen andererseits unterschieden werden (cf. Felfe 2012: 22f.; Burger 2003: 32). Dies gilt umso mehr für die hier analysierten Partikelverben in den bairischen Basisdialekten, bei denen eine Zuordnung zu lexikalischer Transparenz oder Opazität häufig nur schwer aus Publikationen zur Standardsprache abgeleitet werden kann. Allerdings kann der Status einzelner Partikelverben beispielsweise aus der Tilgung von Verbpartikeln empirisch ermittelt werden: Kann die Verbpartikel getilgt werden, ist von transparenten Partikelverben auszugehen, da die dominanten Basisverben die Gesamtbedeutung ohne Bedeutungsverlust selbstständig tragen können. $\mathrm{Zu}$ überprüfen ist, ob opake

\footnotetext{
${ }^{3}$ Die Studie von Schabus (1982) bezieht sich auf die südbairischen Dialekte im Bundesland Kärnten. Für den südmittelbairischen Raum Tirols verzeichnete Küng (1972: 26) wiederum einen Ersatz des Präfixes der- durch zer-.
} 
Partikelverbkonstruktionen aufgrund ihrer engeren semantischen Verbindung den Konjunktiv II häufiger periphrastisch bilden, um die morpho-syntaktische Trennung von Verbpartikel und Basisverb zu umgehen.

\section{Daten und Methoden}

Im folgenden Abschnitt werden das Untersuchungsgebiet und das Informant*innensample (Kapitel 3.1) sowie das Forschungsdesign und die Untersuchungsmethode (Kapitel 3.2) erläutert. Außerdem wird genauer auf die untersuchten Items eingegangen (Kapitel 3.3).

\subsection{Untersuchungsgebiet und Gewährspersonen}

Das Untersuchungsgebiet umfasst das Bundesland Salzburg, das mit dem Westmittelbairischen (im nördlichen Flachgau), dem südmittelbairischen Übergangsgebiet (im südlichen Flachgau sowie dem Tennengau, Pongau und Pinzgau) und dem Südbairischen (im Lungau) von drei größeren Dialektarealen durchzogen wird (cf. Wiesinger 1983). Die sechs Untersuchungsorte der vorliegenden Studie sind entsprechend der Größe dieser Dialektgebiete im Bundesland Salzburg verteilt: Für das Westmittelbairische wurden in einem Untersuchungsort Daten erhoben, das Südmittelbairische ist durch drei und das Südbairische durch zwei Erhebungsorte repräsentiert. Abbildung 1 zeigt die Untersuchungsorte und ihre Lage sowohl in den Bezirken als auch in den Dialektarealen.

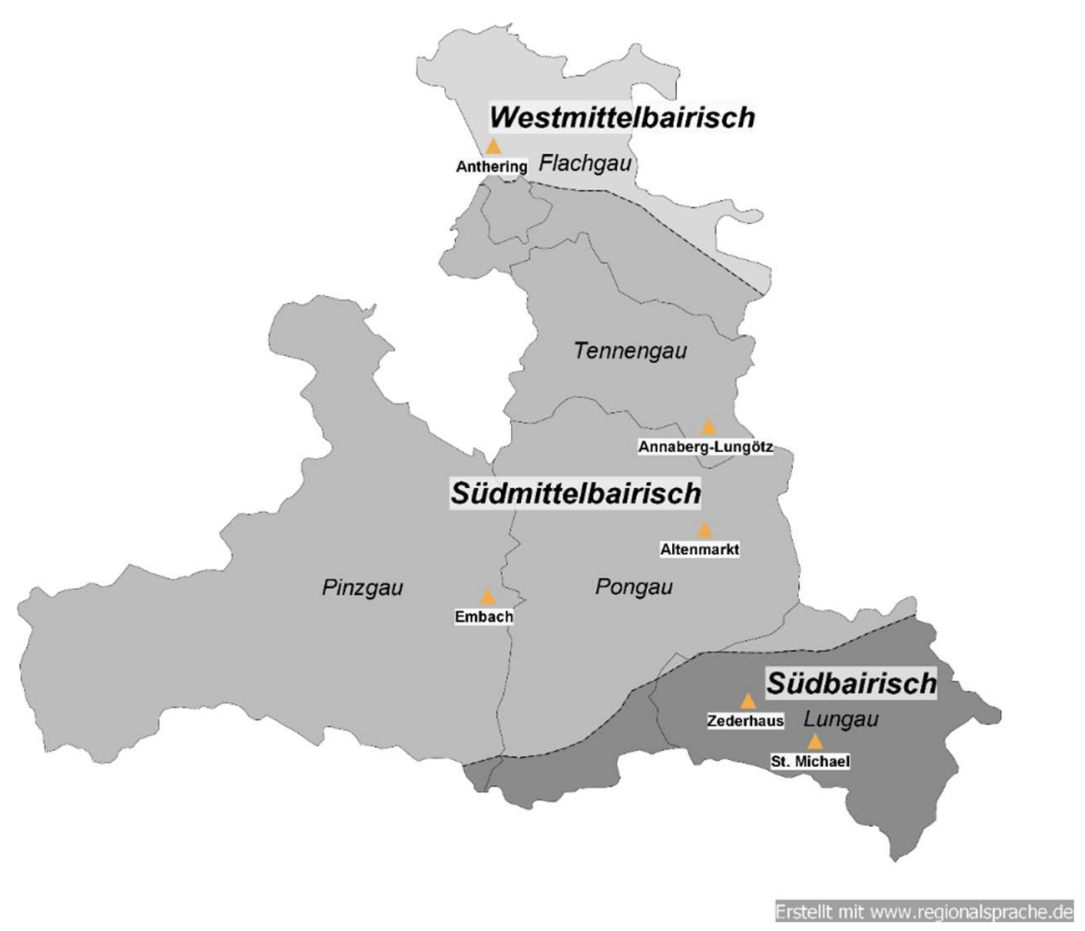

Abbildung 1: Untersuchungsorte und ihre Lage in den Bezirken und Dialektarealen Salzburgs

Tabelle 3 fasst das Gewährspersonensample nach Ortspunkt und Altersgruppe zusammen. In jedem Ort wurden mindestens vier Gewährspersonen (GP) befragt, jeweils zwei jüngere (1831 Jahre; $\varnothing=25$ Jahre, SD = 4,4 Jahre) und zwei ältere (60+ Jahre; $\varnothing=68$ Jahre, SD = 7,1 Jahre). Von den 25 GP sind 15 weiblich und zehn männlich. Zwanzig GP haben keine Matura, 
wobei zwölf einen Pflichtschul- oder Lehrabschluss und acht eine berufsbildende Schule ohne Matura als höchste abgeschlossene Ausbildung angegeben haben.

\begin{tabular}{|l|c|c|c|c|c|c|c|}
\hline Erhebungsort & Anthering & $\begin{array}{c}\text { Annaberg- } \\
\text { Lungötz }\end{array}$ & $\begin{array}{c}\text { Alten- } \\
\text { markt }\end{array}$ & Embach & $\begin{array}{c}\text { Zeder- } \\
\text { haus }\end{array}$ & $\begin{array}{c}\text { Sankt } \\
\text { Michael }\end{array}$ & Gesamt \\
\hline Jüngere GP & 2 & 3 & 2 & 2 & 2 & 2 & 13 \\
\hline Ältere GP & 2 & $2^{4}$ & 2 & 2 & 2 & 2 & 12 \\
\hline Gesamt & 4 & 5 & 4 & 4 & 4 & 4 & 25 \\
\hline
\end{tabular}

Tabelle 3: Verteilung der Gewährspersonen nach Altersgruppe und Ortspunkt

\subsection{Forschungsdesign und Methode}

Da aktuelle Untersuchungen deutliche Alterseffekte zugunsten der Verwendung periphrastischer Varianten bei jüngeren GP aufzeigen (cf. Breuer/Wittibschlager 2020; Vergeiner/Bülow 2022), soll allfälligen Wandeltendenzen auch in dieser Studie mithilfe eines apparent-timeDesigns Rechnung getragen werden.

Die Daten wurden zwischen März und August 2021 indirekt mithilfe eines Fragebogens erhoben, der sich im Hinblick auf die verwendeten Aufgabentypen (Bewertungsaufgaben, Ergänzungsaufgaben) an aktuelleren Syntaxfragebogenstudien orientiert (cf. indirekte Befragungen in SyHD und DiÖ; Fleischer/Kasper/Lenz 2012: 17, 24). Dieses Vorgehen gewährleistet eine gute Kontrolle über die Zielvariablen sowie eine hohe Vergleichbarkeit der gewonnenen Daten. Außerdem konnte so sichergestellt werden, dass einerseits die Partikelverben und andererseits die dazugehörigen einfachen Verben in ausreichender Anzahl in Verbzweitsatzkonstruktionen ${ }^{5}$ elizitiert wurden (siehe Tabelle 4). In einigen Fällen verwendeten die GP dennoch Synonyme für die ausgewählten Verben - diese Belege wurden zwar nicht ausgewertet, jedoch geben sie Aufschluss über die Gebräuchlichkeit der Partikelverben in den bairischen Basisdialekten. Durch passende Situationsbeschreibungen und die Vorgabe von Inhaltsbausteinen konnten zudem homonyme Lesarten bei Verben wie angeben (in diesem Fall ,prahlen') vermieden werden.

Der Fragebogen enthielt drei Aufgabenreihen. Teil 1 bezog sich auf die Erhebung einfacher Verben, die in den Teilen 2 und 3 als Basisverben für die Partikelverbbildungen herangezogen wurden (siehe Kapitel 3.3). Bei den Aufgaben in Teil 1 wurde abgefragt, welche der sechs Konjunktiv-II-Varianten die GP in ihrem Dialekt in den angegebenen Situationen verwenden können (siehe Tabelle 2 in Kapitel 2.1.1). Die in Sätze eingebetteten Varianten wurden hinsichtlich der jeweiligen Dialektregion eingelautet (bzw. dialektalisiert). ${ }^{6}$ Zudem bestand immer auch die Möglichkeit, den gewünschten Satz in einer eigenen Form frei wiederzugeben. Dieser Aufgabentyp ermöglichte Mehrfachantworten, wobei die GP alle Varianten ankreuzen konnten, die in ihrem Dialekt möglich sind. Im Anschluss wurde nach der natürlichsten Variante gefragt,

\footnotetext{
${ }^{4}$ Eine der beiden älteren Gewährspersonen (64 Jahre) ist bis zu ihrem 23. Lebensjahr im benachbarten Ort Sankt Martin am Tennengebirge aufgewachsen, lebt nun aber schon seit 41 Jahren in Annaberg-Lungötz.

5 Verberstsätze wurden zum Zweck der Vergleichbarkeit der Daten ausgeschlossen.

${ }^{6}$ Bei vier der untersuchten einfachen Verben (bringen, geben, lesen, nehmen) ist eine Bildung mit synthetischen Formen ohne -at-Suffix bekannt und mehr oder weniger gebräuchlich (cf. Vergeiner/Bülow 2022), sodass sechs Varianten vorgegeben wurden. Für die übrigen Verben (kaufen, nähen, ziehen, sagen), die keine starken synthetischen Formen aufweisen, wurden entsprechend nur fünf Varianten vorgegeben.
} 
wobei diesmal Mehrfachantworten ausgeschlossen waren. Die möglichen Varianten geben Aufschluss über die Variantenverbreitung bei einzelnen Verben. Für den Vergleich zwischen den einfachen Verben und den entsprechenden Partikelverben wurde jedoch nur die natürlichste Variante herangezogen. ${ }^{7}$

Auch die Aufgaben in Teil 2 beinhalteten Situationsbeschreibungen (siehe Abbildung 2). Die Gewährspersonen wurden angewiesen, die Sätze mithilfe der Inhaltsbausteine in den Klammern zu vervollständigen. Alle Aufgaben der Aufgabenreihe 2 sollten irreale Konditionale elizitieren.

\section{Beispielaufgabe 1 zu abnehmen:}

Nach dem Großeinkauf bittet Ihre Mutter Sie, ihr eine Einkaufstasche abzunehmen. Sie haben aber selbst alle Hände voll und können keine weitere Tasche tragen. Sie sagen deshalb zu Ihrer Mutter, dass Sie ihr eigentlich gerne eine Tasche abnehmen, aber leider selbst keine Hand frei haben.

[dir] [gerne] [eine Tasche] [abnehmen]

I , oba i hob söwa koa Hond frei.

Abbildung 2: Beispielaufgabe zu abnehmen in der Aufgabenreihe 2

Im dritten Teil des Fragebogens wurden die Gewährspersonen dazu angehalten, potentielle Konditionalsätze in der 1.Ps.Sg. im Konjunktiv II anhand vorgegebener Inhaltsbausteine zu bilden (siehe Abbildung 3).

Beispielaufgabe 2 zu annähen:

mehr Zeit haben - den Knopf annähen $\rightarrow$

Abbildung 3: Beispielaufgabe zu annähen in der Aufgabenreihe 3

\subsection{Items}

Für die vorliegende Studie wurden 15 Partikelverben sowie die dazugehörigen acht Basisverben untersucht. Die acht Basisverben entsprechen einfachen Verben, die auch in der Untersuchung von Vergeiner/Bülow (2022) abgefragt wurden. Die daraus gebildeten Partikelverben sind im Dialekt der Erstautorin dieses Beitrags geläufig. Dieser Eindruck konnte durch Pretests bestätigt werden.

Mit Ausnahme von nähen wurde jedes einfache Verb mit zwei verschiedenen Partikeln linkserweitert (siehe Tabelle 4). Jedes Partikelverb wurde zweifach getestet (in Teil 2 und 3 des Fragebogens), um mögliche Gebrauchsunterschiede in verschiedenen Konstruktionstypen (z. B. irreales/potentielles Konditionalgefüge) feststellen zu können (siehe Kapitel 3.2). Vergleicht man allerdings die Ergebnisse aus Teil 2 und 3 des Fragebogens, ergeben sich keine statistisch signifikanten Unterschiede hinsichtlich der Konjunktiv-II-Variation $\left(\chi^{2}(1)=1,246\right.$;

\footnotetext{
${ }^{7}$ Vereinzelt wurde auf die Angabe der natürlichsten Variante verzichtet, jedoch auch im Mehrfachantworten-Setting nur eine Variante angekreuzt. Diese Variante wurde dann als natürlichste Variante gewertet.
} 
$\mathrm{p}=.264=\mathrm{n} . \mathrm{s}$.), weshalb die Daten aus Teil 2 und 3 für die folgende Analyse zusammengefasst wurden.

\begin{tabular}{|c|c|c|c|}
\hline Einfache Verben & Gültige Belege & Partikelverb & Gültige Belege \\
\hline \multirow[t]{2}{*}{ ziehen } & \multirow[t]{2}{*}{22} & aufziehen & 45 \\
\hline & & anziehen & 36 \\
\hline \multirow[t]{2}{*}{ sagen } & \multirow[t]{2}{*}{23} & einsagen & 40 \\
\hline & & ansagen & 47 \\
\hline \multirow[t]{2}{*}{ nehmen } & \multirow[t]{2}{*}{23} & abnehmen & 47 \\
\hline & & aufnehmen & 41 \\
\hline \multirow[t]{2}{*}{ kaufen } & \multirow[t]{2}{*}{23} & einkaufen & 34 \\
\hline & & abkaufen & 48 \\
\hline nähen & 23 & annähen & 45 \\
\hline \multirow[t]{2}{*}{ lesen } & \multirow[t]{2}{*}{21} & ablesen & 33 \\
\hline & & einlesen & 42 \\
\hline \multirow[t]{2}{*}{ geben } & \multirow[t]{2}{*}{23} & angeben & 43 \\
\hline & & aufgeben & 38 \\
\hline \multirow[t]{2}{*}{ bringen } & \multirow[t]{2}{*}{22} & abbringen & 37 \\
\hline & & einbringen & 25 \\
\hline Belege gesamt & $\mathrm{n}=180$ & Belege gesamt & 601 \\
\hline
\end{tabular}

Tabelle 4: Anzahl der Belege je Verb

Für das Partikelverb einbringen wurde mit der Phrase das Heu einbringen zur Erhebung ein landwirtschaftlicher Funktionsbereich ausgewählt. Die niedrigen Belegzahlen von einbringen sind der häufigeren Verwendung von synonymen Umschreibungen wie das Heu hineintun oder heign (,heuen') geschuldet. Synonyme und Periphrasen bspw. mit den Modalverben mögen und können wurden nicht in die Auswertung einbezogen. Bei einkaufen wurde häufiger die Partikel weggelassen und stattdessen das einfache Verb kaufen verwendet - auch diese Belege konnten für die vorliegende Studie nicht ausgewertet werden, jedoch geben sie Aufschluss über den lexikalischen Status des Partikelverbs und den geringen Grad an semantischer Modifikation des Basisverbs.

\section{$4 \quad$ Ergebnisse}

Im folgenden Abschnitt werden die zentralen Ergebnisse der Studie präsentiert. Einleitend werden die allgemeinen Ergebnisse der Studie vorgestellt (Kapitel 4.1). Darauf folgt die Präsentation der Ergebnisse im Hinblick auf innersprachliche (Kapitel 4.2), sprachgeografische (Kapitel 4.3) und soziolinguistische Steuerungsfaktoren (Kapitel 4.4).

\subsection{Allgemeine Ergebnisse}

In der vorliegenden Studie werden sechs Konjunktiv-II-Varianten unterschieden: Synthetische Varianten ohne Suffigierung oder mit -at-Markierung, periphrastische Varianten mit tun- oder würde-Auxiliar sowie die jeweiligen periphrastischen Hybridformen mit würde oder tun und - 
at-Suffix (siehe Tabelle 2 in Kapitel 2.1.1). Abbildung 4 zeigt die Distribution dieser sechs Varianten bei den acht einfachen Verben.

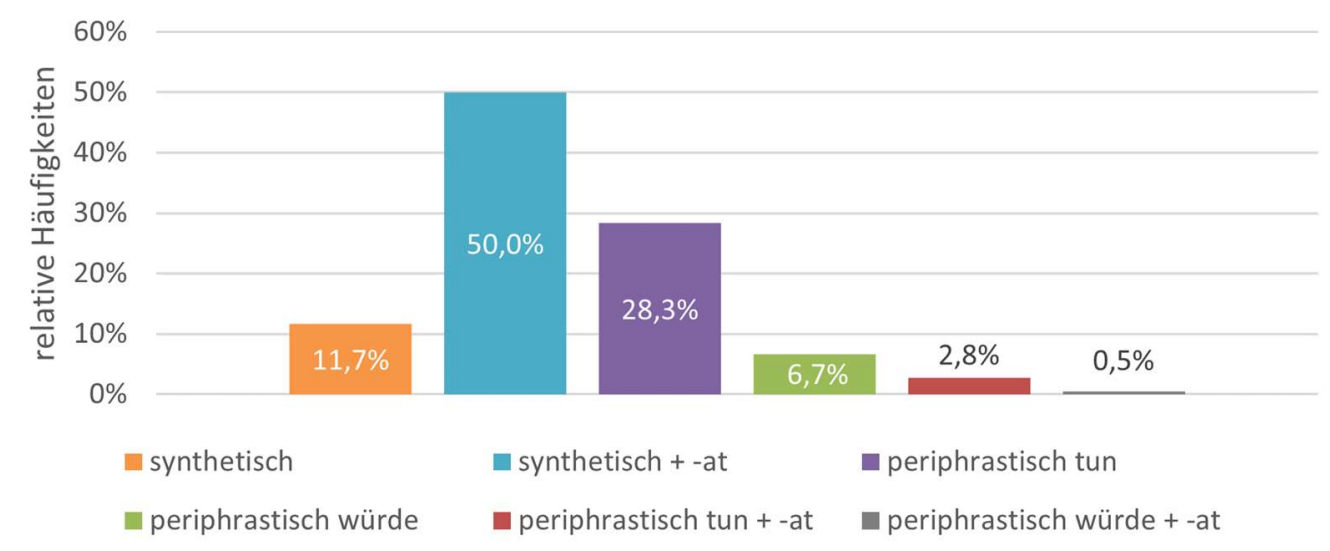

Abbildung 4: Relative Häufigkeiten (in \%) der Konjunktiv-II-Varianten im Hinblick auf die untersuchten einfachen Verben $(n=180)$

Insgesamt sind synthetische Varianten $(\mathrm{n}=111$; entspricht $61,7 \%)$ bei den einfachen Verben häufiger belegt als periphrastische Varianten $(n=69$; entspricht 38,3\%). Unter den synthetischen Varianten dominiert die Form mit -at-Suffix deutlich gegenüber der Variante ohne -at. Eine klare Präferenz für die Form mit tun-Auxiliar ohne -at-Suffix ist unter den periphrastischen Varianten erkennbar; die einfache würde-Periphrase sowie hybride Varianten mit Auxiliar $+-a t$ sind deutlich seltener dokumentiert (siehe Abbildung 4).

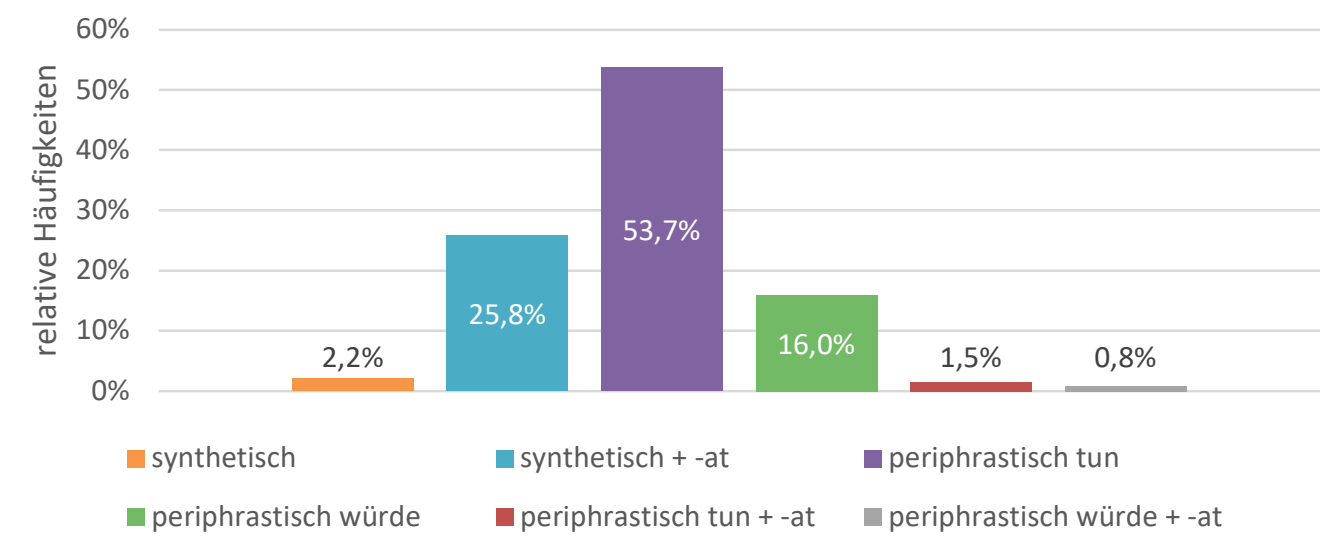

Abbildung 5: Relative Häufigkeiten (in \%) der Konjunktiv-II-Varianten im Hinblick auf die untersuchten Partikelverben $(\mathrm{n}=601)$

Die Daten zu den untersuchten Partikelverben zeigen eine gänzlich andere Verteilung der Konjunktiv-II-Varianten: Periphrastische Formen überwiegen mit 72\% $(n=433)$ klar (siehe Abbildung 5). 53,7\% $(\mathrm{n}=323)$ der Gesamtbelege entfallen dabei auf die einfache tun-Periphrase, die damit eindeutig die präferierte Variante darstellt. Synthetische Formen wurden mit 28\% $(\mathrm{n}=168)$ deutlich seltener gebildet als periphrastische, wobei die Variante mit -at-Suffix ( $n=155$; entspricht 25,8\%) klar bevorzugt wurde. Die nicht-suffigierte synthetische Variante ist hier, wie auch die beiden hybriden periphrastischen Formen mit Auxiliar und -at-Suffix, äußerst selten belegt. 
Im Vergleich zwischen den einfachen Verben und den Partikelverben zeichnet sich somit ein auffälliger Unterschied zwischen dem synthetischen und periphrastischen Bildungstyp ab: Während bei den einfachen Verben synthetische Formen dominieren, überwiegen periphrastische Varianten bei den Partikelverben.

\subsection{Innersprachliche Faktoren}

Um verbspezifische Unterschiede aufzudecken, wurden sowohl die einfachen Verben als auch die Partikelverben gesondert untersucht. Abbildung 6 zeigt, wie sich die Konjunktiv-II-Varianten auf die einzelnen einfachen Verben verteilen.

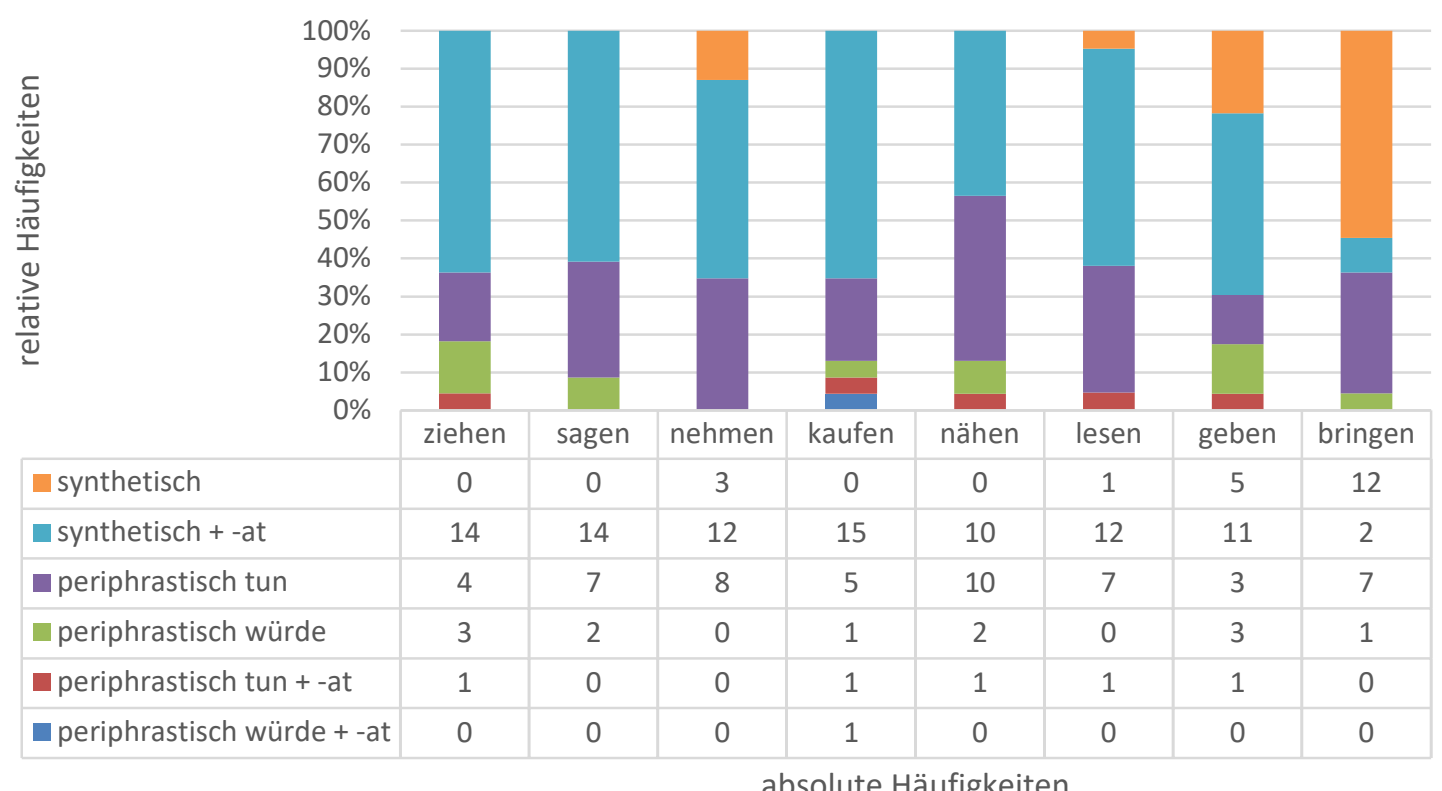

\section{Abbildung 6: Relative (in \%) und absolute Häufigkeiten $(n=180)$ der Konjunktiv-II-Varianten im Hinblick auf die einfachen Verben}

Bei sieben der acht untersuchten einfachen Verben überwiegen synthetische Formen, nur nähen erscheint häufiger mit periphrastischen Formen. In der Studie von Vergeiner/Bülow (2022) zeichnet sich bei den Verben ziehen, sagen, nehmen und kaufen im Bundesland Salzburg eine Präferenz für die synthetische Variante mit -at-Suffix ab, welche in dieser Studie bestätigt werden kann. Für die Verben nähen und lesen dokumentieren Vergeiner/Bülow (2022) eine verstärkte Tendenz zur periphrastischen Form mit tun-Auxiliar ohne -at-Suffix, wobei im Bundesland Salzburg auch bei diesen Verben etwas häufiger als in den meisten anderen Bundesländern die synthetische Variante mit -at-Suffix verwendet wird. Eine Tendenz zu periphrastischen Formen ist in dieser Studie bei nähen, nicht aber bei lesen erkennbar.

Die Verben bringen und geben sind bei Vergeiner/Bülow (2022) durch eine signifikant häufigere Verwendung der starken synthetischen Konjunktiv-II-Form ohne -at vor allem im Westen und im Zentrum Österreichs - Salzburg eingeschlossen - gekennzeichnet. Für das Verb bringen kann diese Tendenz bestätigt werden: In 54,5\% $(\mathrm{n}=12)$ aller Belege wurde bringen mit Stammvokalwechsel ohne -at dokumentiert. Für das Verb geben kann von allen Verben der zweithöchste Anteil an der nicht-suffigierten synthetischen Form $(\mathrm{n}=5$; entspricht 21,7\%) verzeichnet werden, jedoch überwiegt die Variante mit -at-Suffix $(n=11$; entspricht 47,8\%). 
Wie schon in Kapitel 4.1 deutlich geworden ist, zeigen die Partikelverben eine stärkere Tendenz zu periphrastischen Formen. Das illustriert auch Abbildung 7, in der die Verteilung der Konjunktiv-II-Varianten je Basisverb zusammengefasst ist. Bei allen Basisverben überwiegt die periphrastische Form mit tun-Auxiliar klar. Die einfache würde-Periphrase kommt mit 12,3\% (-ziehen, $\mathrm{n}=10$ ) bis 19,4\% (-bringen, $\mathrm{n}=12$ ) bei allen Basisverben in vergleichbar hohem Ausmaß vor. Synthetische Formen erscheinen am häufigsten bei Bildungen mit dem Basisverb -kaufen ( $\mathrm{n}=28$; entspricht 34,1\%) und am seltensten bei solchen mit dem Basisverb -geben ( $\mathrm{n}$ $=13$; entspricht $16 \%$ ).

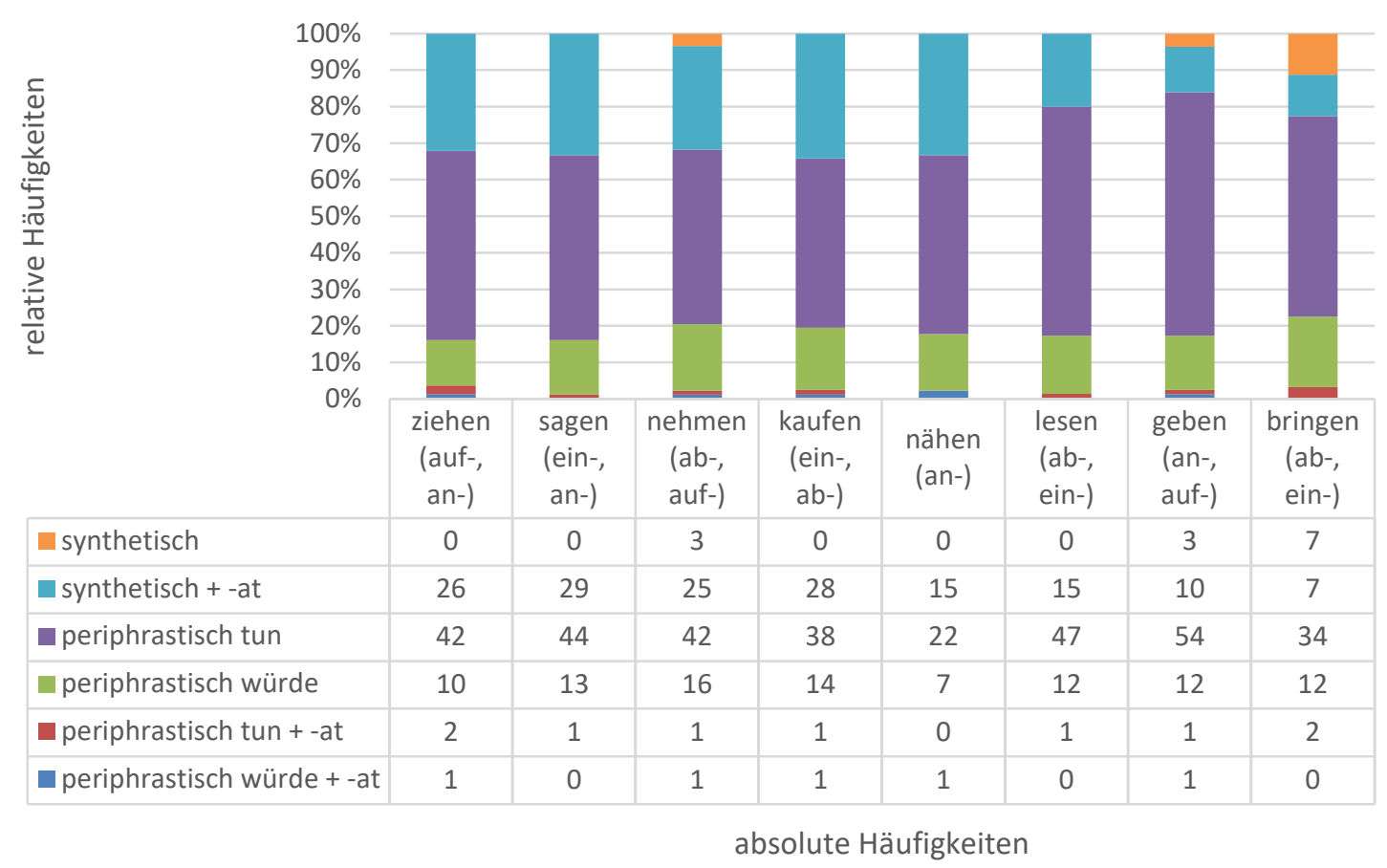

\section{Abbildung 7: Relative (in \%) und absolute Häufigkeiten (n=601) der Konjunktiv-II-Varianten im Hinblick auf die Basisverben der Partikelverbbildungen}

Mit 84\% $(n=68)$ wurde -geben am häufigsten mit periphrastischen Varianten realisiert. Zum Vergleich: Für das einfache Verb geben sind nur 7 (=30,4\%) periphrastische Bildungen dokumentiert. Zwischen dem einfachen Verb nähen (56,5\% periphrastische Bildungen) und den Partikelverbbildungen mit -nähen (66,7\% periphrastische Bildungen) gibt es hingegen nur geringe Unterschiede mit Blick auf die Verwendung periphrastischer Varianten. Zwischen dem einfachen Verb nähen und den Partikelverbbildungen mit -nähen zeigt sich entsprechend kein statistisch signifikanter Zusammenhang hinsichtlich der Gebrauchshäufigkeit synthetischer und periphrastischer Varianten (siehe Tabelle 5). Wie Tabelle 5 zeigt, bestehen allerdings für die übrigen Verben statistisch signifikante Zusammenhänge zwischen dem Vorkommen synthetischer und periphrastischer Konjunktiv-II-Varianten einerseits und der Verwendung als einfache Verben oder Basisverben von Partikelverbkonstruktionen andererseits. 


\begin{tabular}{|c|c|c|c|}
\hline $\begin{array}{c}\text { Einfaches Verb*Basis- } \\
\text { verb der Partikelverbbil- } \\
\text { dung }\end{array}$ & $\mathbf{n}$ & $\chi^{2}$-Test & Effektstärke \\
\hline ziehen*-ziehen $^{*}$ & 103 & $\chi^{2}(1)=7,244 ; p<.007^{* *}$ & Cramer's V $=.265$ \\
\hline sagen*-sagen $^{*}{ }^{*}$-sehmen*-nehmen & 110 & $\chi^{2}(1)=5,793 ; p<.016^{*}$ & Cramer's V $=.229$ \\
\hline kaufen*-kaufen $^{*}$ & 111 & $\chi^{2}(1)=8,571 ; p<.003^{* *}$ & Cramer's V $=.278$ \\
\hline nähen*-nähen & 68 & $\chi^{2}(1)=7,171 ; p<.007^{* *}$ & Cramer's V $=.261$ \\
\hline lesen*-lesen & 96 & $\chi^{2}(1)=.674 ; p=.412=$ n. s. & - \\
\hline geben*-geben & 104 & $\chi^{2}(1)=13,945 ; p<.000^{* * *}$ & Cramer's V $=.381$ \\
\hline bringen*-bringen & 84 & $\chi^{2}(1)=12,317 ; p<.000^{* * *}$ & Cramer's V $=.383$ \\
\hline
\end{tabular}

Tabelle 5: Statistisch signifikante Zusammenhänge zwischen dem Gebrauch synthetischer und periphrastischer Konjunktiv-II-Varianten und den untersuchten Verben als einfache Verben oder Basisverben von Partikelverbkonstruktionen (Gruppierungen: periphrastisch = Formen mit tun- und würde-Auxiliar sowie die jeweiligen Hybridformen mit den Auxiliaren inkl. -at; synthetisch = Formen ohne und mit -at-Suffix)

Zwischen den einfachen Verben und den Basen der Partikelverben (-)ziehen, (-)sagen, (-)nehmen und (-)kaufen zeigt sich ein schwacher statistischer Zusammenhang hinsichtlich des Gebrauchs synthetischer und periphrastischer Konjunktiv-II-Formen (Cramer's V < 0,3). Einen mittleren Effekt ergibt die Berechnung der Effektstärke beim Vergleich der einfachen Verben lesen, bringen und geben mit den Basisverben (-)lesen, (-)bringen und (-)geben (Cramer's $\mathrm{V}<$ $0,5)$.

Um verbspezifische Unterschiede bei den Partikelverben aufzudecken, müssen auch diese gesondert in den Blick genommen werden. Abbildung 8 zeigt die einzelnen Partikelverben absteigend gereiht nach den relativen Gebrauchsfrequenzen synthetischer Konjunktiv-II-Varianten.

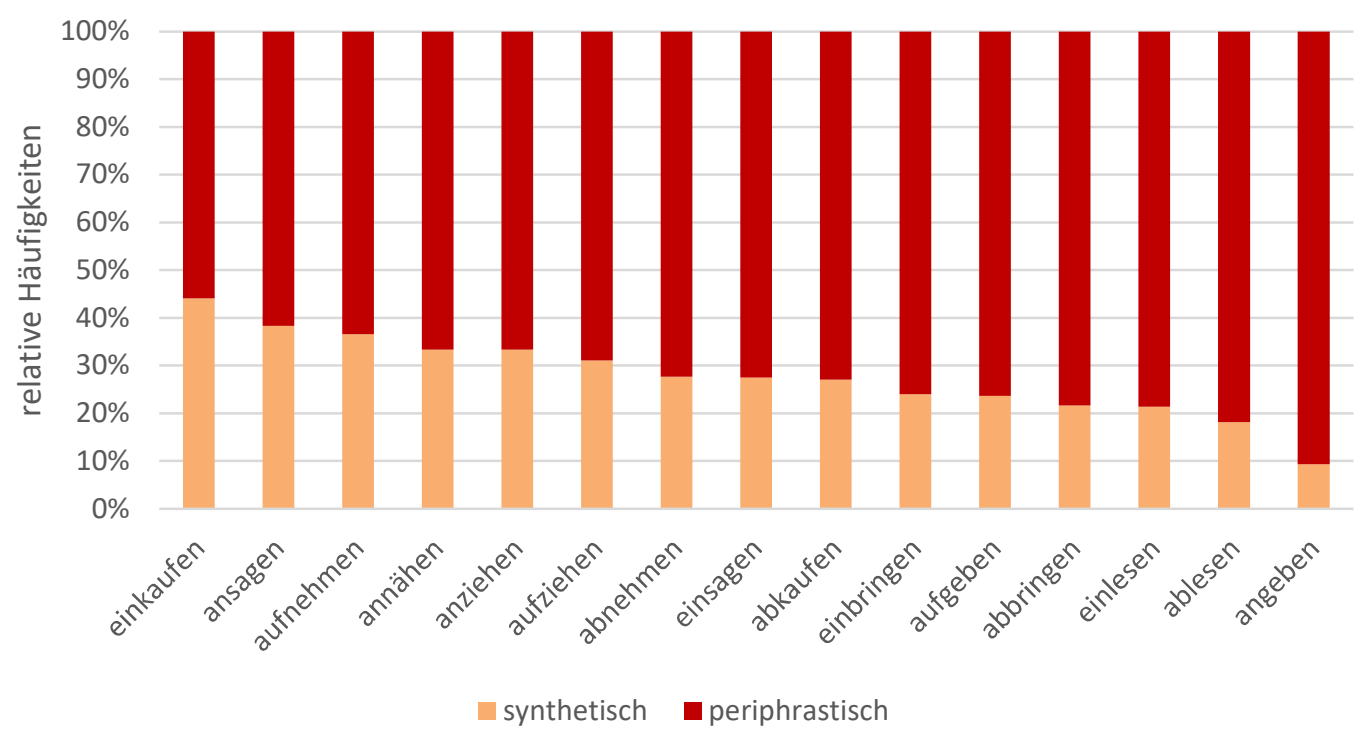

Abbildung 8: Relative Häufigkeiten (in \%) der Konjunktiv-II-Varianten im Hinblick auf die einzelnen untersuchten Partikelverben $(n=601)$

Am häufigsten wurde einkaufen synthetisch realisiert ( $\mathrm{n}=15$; entspricht 44,1\%), am seltensten angeben ( $\mathrm{n}=4$; entspricht 9,3\%). Auffallend sind die Unterschiede zwischen verschiedenen Partikelverben mit demselben Basisverb: einkaufen ( $\mathrm{n}=15$; entspricht 44,1\%) wird 
beispielsweise deutlich öfter mit synthetischen Konjunktiv-II-Varianten gebildet als abkaufen ( $n=13$; entspricht 27,1\%). Bei beiden Partikelverben handelt es sich um lexikalisch transparente Bildungen. Ein bedeutender Unterschied liegt aber in der fakultativen Verwendung der Verbpartikel bei einkaufen: In vielen Belegen ( $n=15$; entspricht 30,6\% der Gesamtbelege), die in die Berechnungen zur Konjunktiv-II-Bildung nicht eingeflossen sind, wurde anstelle des Partikelverbs das einfache Verb kaufen gebildet. Bei einkaufen führt die Verbpartikel offenbar nur zu einer schwachen semantischen Modifikation des Basisverbs (siehe Kapitel 2.2.1).

Beim Partikelverb angeben (,prahlen'), das nur in 9,3\% $(n=4)$ der Fälle mit synthetischen Konjunktiv-II-Varianten erscheint, handelt es sich um ein intransitives Verb, das zudem (dialektal wie standardsprachlich) als semantisch opak gelten kann. ${ }^{8}$ Dass opake Partikelverbkonstruktionen seltener als lexikalisch transparente Bildungen synthetisch realisiert werden, ist aber keinesfalls für jedes hier analysierte Partikelverb zu veranschlagen: So handelt es sich etwa bei einlesen und ablesen um transparente Partikelverbkonstruktionen, die jedoch nach angeben (,prahlen`) am häufigsten periphrastisch realisiert sind. Mögliche Erklärungen für verbspezifische Unterschiede werden in Kapitel 5 eingehender diskutiert.

\subsection{Sprachgeografische Faktoren}

Abbildung 9 illustriert die areale Verteilung der synthetischen und periphrastischen Varianten bei den untersuchten Partikelverben sowie einfachen Verben. Der äußere Kreis zeigt dabei die Verteilung beider Bildungsstrategien bei den einfachen Verben, der innere bei den Partikelverben.

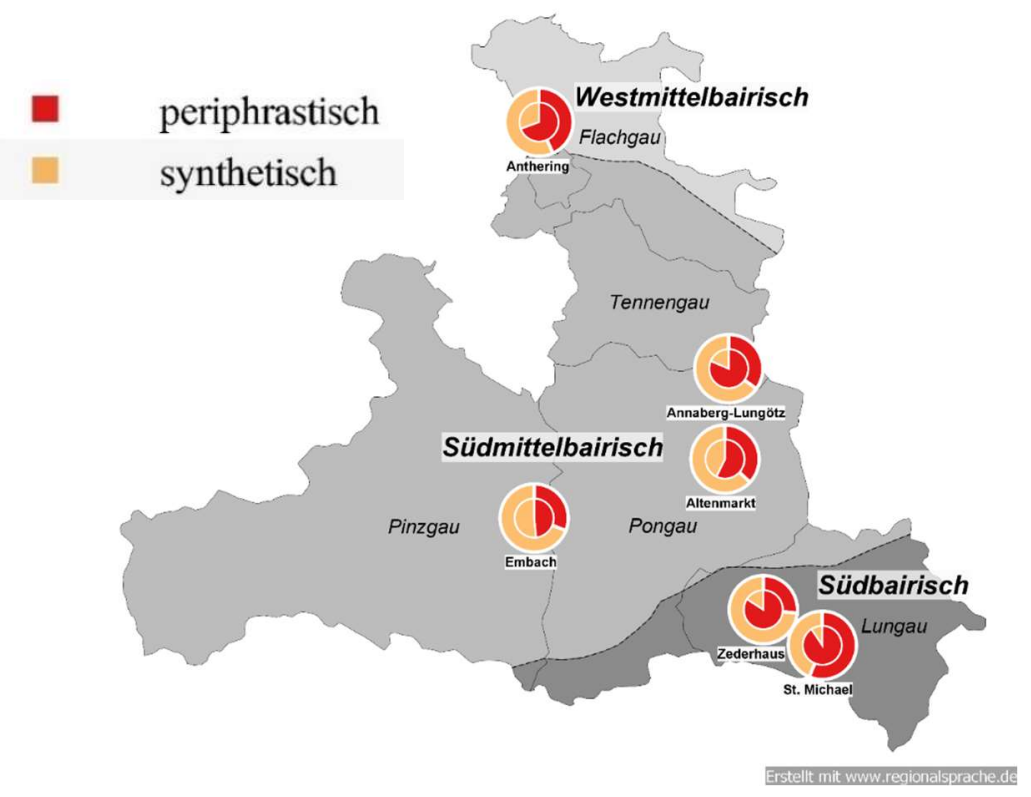

Abbildung 9: Areale Verteilung synthetischer und periphrastischer Varianten bei einfachen Verben $(n=180$, äußerer Kreis) und Partikelverben $(n=601$, innerer Kreis)

\footnotetext{
${ }^{8}$ Durch die vorgegebene Situationsbeschreibung bzw. die Anweisung zur Produktion einer Gesamtkonstruktion mit angeben (,prahlen') anhand vorgegebener Inhaltsbausteine ist eine Bedeutungsverwechslung mit angeben ,mitteilen` ausgeschlossen. Dass die Bedeutung ,prahlen` den Gewährspersonen geläufig ist, belegen hohe Gebrauchsfrequenzen für das Partikelverb und eine äußerst seltene Verwendung von Synonymen wie z. B. aufmoana ,aufmeinen'.
} 
Mit der Ausnahme von St. Michael (Lungau) dominieren in jedem Erhebungsort bei den einfachen Verben synthetische Varianten. Im Hinblick auf die Partikelverben wurden in jedem Untersuchungsort periphrastische Formen präferiert - außer in Embach (Pinzgau), wo synthetische Varianten knapp überwiegen. Alle Erhebungsorte haben zudem gemeinsam, dass sich für die Partikelverben stets ein höherer Anteil an periphrastischen Varianten zeigt als bei den einfachen Verben. Während in Embach (Pinzgau) allerdings nur ein geringer Unterschied besteht (17 Prozentpunkte), ist die Differenz in Zederhaus (Lungau) mit 57 Prozentpunkten mehr als dreimal so hoch ausgeprägt. Aus den Daten lassen sich jedoch keine signifikanten sprachgeografischen Unterschiede erkennen.

\title{
4.4 Soziolinguistische Faktoren
}

Abbildung 10 zeigt die Realisierungsfrequenzen der Konjunktiv-II-Varianten bei den einfachen Verben und Partikelverben für die beiden Altersgruppen alt und jung.

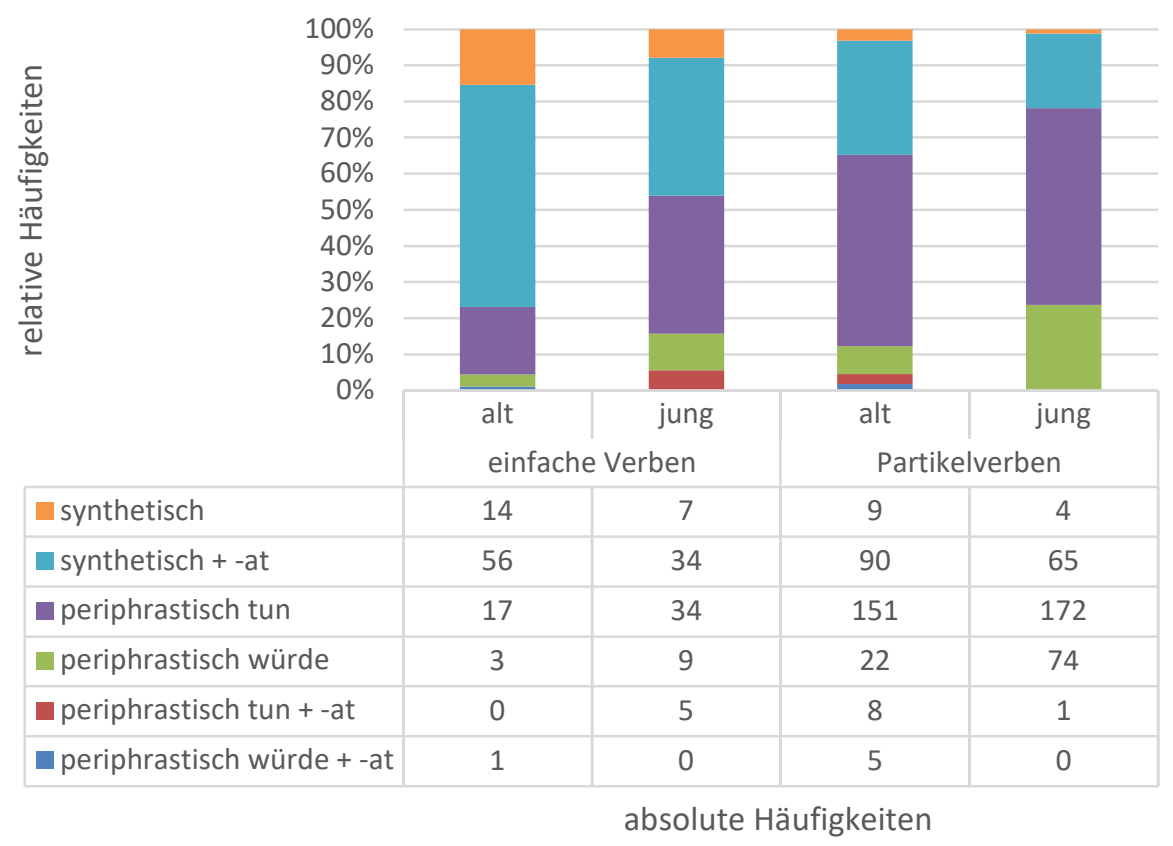

\begin{abstract}
Abbildung 10: Relative (in \%) und absolute Häufigkeiten der Konjunktiv-II-Varianten je Verbgruppe (einfache Verben $(n=180)$ vs. Partikelverben $(n=601))$ und Altersgruppe (alt und jung)
\end{abstract}

Bei beiden Verbgruppen ist eine häufigere Verwendung von periphrastischen Varianten und eine damit verbundene seltenere Verwendung synthetischer Varianten bei den jungen GP auffällig. Ein statistisch signifikanter Zusammenhang zwischen der Verwendung synthetischer und periphrastischer Konjunktiv-II-Varianten einerseits und dem Alter der Gewährspersonen (alt/jung) andererseits besteht sowohl bei den einfachen Verben $\left(\chi^{2}(1)=18,122 ; p<.000 * * *\right.$ (Cramer's V =.317)) als auch bei den Partikelverben $\left(\chi^{2}(1)=12,385 ; p<.000 * * *\right.$ (Cramer's $\mathrm{V}=.144))$.

Während synthetische Varianten bei älteren GP bei einfachen Verben noch dominieren (in $76,9 \%$ der Fälle), überwiegen bei den jüngeren GP bereits die periphrastischen Varianten (in $53,9 \%$ der Fälle). Bei den Partikelverben dominieren die periphrastischen Varianten sowohl bei den älteren (in 65,3\% der Fälle) als auch bei den jüngeren GP (in 78,2\% der Fälle), wobei der 
Anteil an periphrastischen Varianten bei den jüngeren GP nochmal um 13 Prozentpunkte höher ist. Außerdem fällt auf, dass die jüngeren GP sowohl bei den einfachen Verben als auch bei den Partikelverben öfter die einfache würde-Periphrase verwenden als die älteren GP. Die jüngeren GP gebrauchen die Variante mit würde-Auxiliar ohne -at-Suffix insgesamt mehr als dreimal so häufig wie die älteren GP. Die eindeutig präferierte periphrastische Variante stellt dennoch mit Abstand die einfache tun-Periphrase dar.

Die Ergebnisse dieser Studie belegen einerseits einen starken Einfluss der Variable Alter auf die Konjunktiv-II-Bildung, im Vergleich zwischen den beiden Verbgruppen zeigt sich andererseits, dass sowohl bei den einfachen Verben als auch bei den Partikelverben jeweils periphrastische Varianten von den jüngeren GP häufiger verwendet werden.

Im Gegensatz zur Variable Alter kann für die Variable Geschlecht kein Effekt nachgewiesen werden, wie Abbildung 11 zeigt.

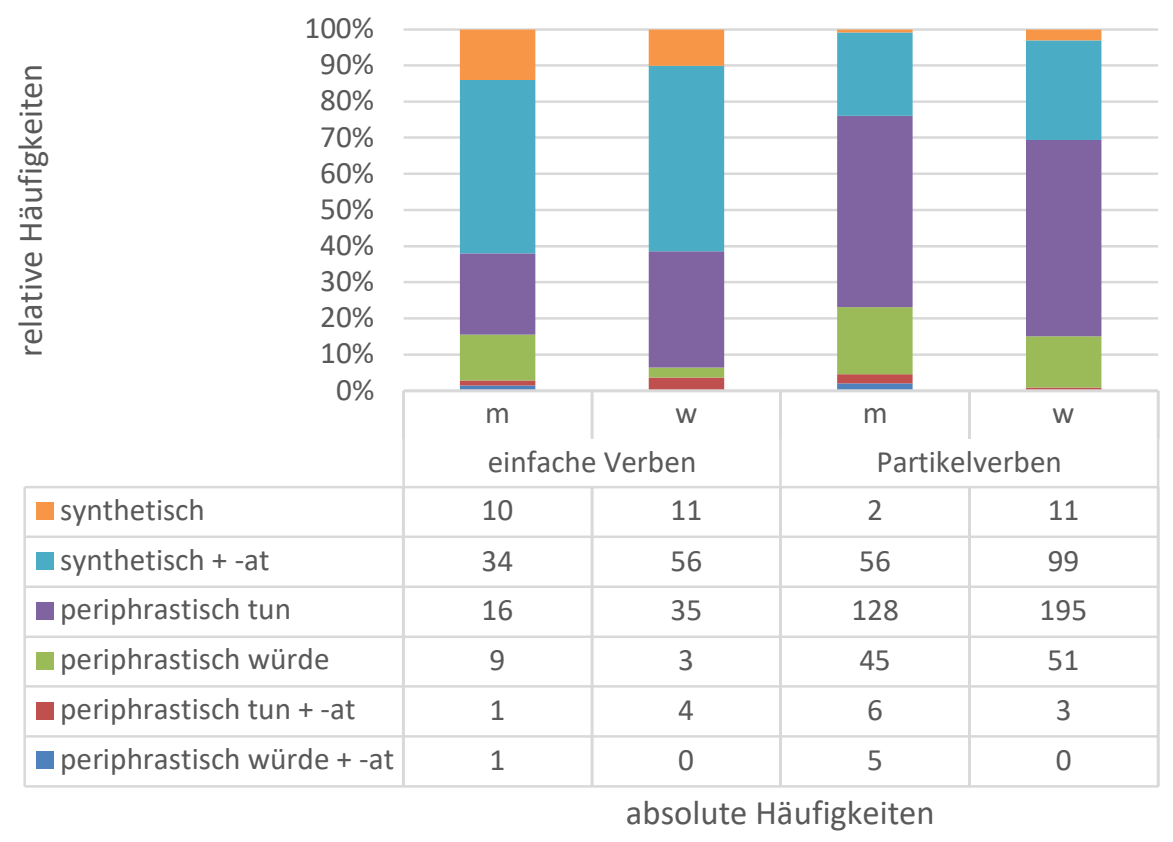

Abbildung 11: Relative (in \%) und absolute Häufigkeiten der Konjunktiv-II-Varianten je Verbgruppe (einfache Verben $(n=180)$ vs. Partikelverben $(n=601))$ und Geschlecht $(m=$ männlich, $w=$ weiblich)

Bei beiden Geschlechtern überwiegen bei den einfachen Verben synthetische Varianten und bei den Partikelverben periphrastische Varianten in vergleichbarem Umfang. Ein statistisch signifikanter Zusammenhang zwischen dem Gebrauch synthetischer und periphrastischer Varianten und dem Geschlecht der Gewährspersonen (männlich/weiblich) besteht weder bei den einfachen Verben $\left(\chi^{2}(1)=.005 ; p=.946=\right.$ n. s. $)$ noch bei den Partikelverben $\left(\chi^{2}(1)=3.197 ; p=.074\right.$ =n. s.). ${ }^{9}$

\footnotetext{
${ }^{9}$ Auffällig ist eine stärkere Tendenz zur Verwendung der periphrastischen Variante mit würde-Auxiliar bei männlichen GP, die jedoch interindividuellen Unterschieden geschuldet ist: Von allen verzeichneten einfachen würdePeriphrasen bei männlichen GP entfallen 44,4\% $(n=4)$ bei den einfachen Verben und 48,9\% $(n=22)$ bei den Partikelverben auf eine einzige männliche GP.
} 


\section{Diskussion}

Die Ergebnisse in Kapitel 4 haben gezeigt, dass die Konjunktiv-II-Bildung bei Partikelverben in den Salzburger Dialekten durch innersprachliche, sprachgeografische und soziolinguistische Faktoren beeinflusst wird. In diesem Kapitel werden die Ergebnisse ausführlicher diskutiert. Dabei rücken die Unterschiede bei der Konjunktiv-II-Bildung zwischen Partikelverben und einfachen Verben sowie anschließend zwischen einzelnen Partikelverben in den Fokus der Diskussion. Zuletzt wird näher auf die Rolle sprachgeografischer und soziolinguistischer Steuerungsfaktoren eingegangen.

Kapitel 4.1 hat gezeigt, dass Partikelverben signifikant häufiger mit periphrastischen Konjunktiv-II-Varianten erscheinen als einfache Verben. Eine Erklärung für diesen Befund bietet die in Kapitel 1 beschriebene Tatsache, dass die synthetische Konjunktiv-II-Bildung eine morphologische Distanzstellung von Verbpartikel und Basisverb erfordert, wohingegen die periphrastische Konjunktiv-II-Bildung eine Kontaktstellung erlaubt. Entsprechend der Annahme, dass Sprecher*innen dazu tendieren, „geistig eng Zusammengehöriges“ (im vorliegenden Fall: Verbpartikel und Basisverb) adjazent im Satz zu positionieren (Behaghel 1932: 4), stellt das Präferenzgesetz der Nähe eine mögliche Erklärung für diesen Befund dar.

Unter kognitiven Gesichtspunkten dürften periphrastische Konjunktiv-II-Formen eine einfachere Prozessierung erlauben, vor allem bei Partikelverben, bei denen der semantische Gehalt nur aus der Kombination von Verbpartikel und Basisverb (sowie ggf. dem syntaktischen Kontext) erschlossen werden kann (siehe Kapitel 2.2; cf. Felfe 2012: 22). Stehen Verbpartikel und Basisverb in Kontaktstellung, wird eine einfachere Identifikation der Gesamtbedeutung eines Partikelverbs ermöglicht. Mit dem Auxiliar fungiert außerdem eine dritte, gewissermaßen „neutrale“ Komponente als Träger der grammatischen Information Modus.

In Kapitel 4.2 wurde gezeigt, dass für einzelne Verben jedoch unterschiedlich starke Zusammenhänge zwischen der morphologischen Bildung (einfaches Verb oder Basisverb bei einem Partikelverb) und dem Vorkommen synthetischer oder periphrastischer Konjunktiv-II-Varianten bestehen. Diese Unterschiede scheinen u. a. damit zusammenzuhängen, ob das zugrundeliegende (Basis-)Verb seine synthetische Variante regulär (i. e. mit Affigierung) oder irregulär (i. e. mit Stammmodifikation) bildet: So bestehen etwa zwischen bringen (einfaches Verb) und -bringen (Basisverb bei einem Partikelverb) stärkere Zusammenhänge mit der Realisierung synthetischer oder periphrastischer Varianten als bei den meisten anderen Verben. Als einfaches Verb ist bringen zu 54,5\% $(\mathrm{n}=12)$ in der nicht-suffigierten synthetischen Variante bracht belegt, die beim Basisverb -bringen mit 11,3\% $(\mathrm{n}=7)$ bemerkenswert selten vorkommt. Offensichtlich steht diese irreguläre Form (mit Änderungen im Stammvokal und Konsonantismus) für die Flexion des Basisverbs -bringen nur eingeschränkt zur Verfügung (eine Rolle könnte hier auch spielen, dass die abgefragten Partikelverben einbringen bzw. abbringen stark lexikalisiert sind, siehe unten). Die synthetische Variante (-)bringat mit -at-Suffix am Präsensstamm wäre eine morphologisch reguläre Alternativkonstruktion, die erstens den Präsensstamm erhält und zweitens mit dem -at-Suffix eine unverkennbare Konjunktivmarkierung aufweist. Diese Variante scheint bei (-)bringen in den Dialekten Salzburgs jedoch nicht gebräuchlich zu sein.

Anders verhält es sich bei (-)ziehen, (-)nehmen, (-)sagen und (-)kaufen. Hier ist der Zusammenhang zwischen dem Gebrauch als einfaches Verb oder Partikelverb und der Verwendung 
synthetischer oder periphrastischer Formen schwächer ausgeprägt. Dies könnte damit erklärt werden, dass bei (-)ziehen, (-)nehmen, (-)sagen und (-)kaufen - wenn synthetische Varianten verwendet werden - die morphologisch reguläre Bildung mit dem -at-Suffix dominiert (cf. für einen ähnlichen Befund Vergeiner/Bülow 2022). Auszugehen ist davon, dass sich Partikelverben (z. B. einkaufen), deren Basisverben (z. B. (-)kaufen) als einfache Verben (z. B. kaufen) im Konjunktiv II häufiger synthetisch mit -at suffigiert werden, leichter in das synthetische Bildungsschema integrieren lassen als Partikelverben, deren Basisverben als einfache Verben den Konjunktiv II bevorzugt irregulär (i. e. mit Stammmodifikation) bilden (z. B. (-)bringen, bringen). Mit Vergeiner/Bülow (2022) lässt sich dabei u. a. auf die Kriterien der Morphologischen Natürlichkeitstheorie verweisen, denen die synthetische Variante mit -at unter allen Konjunktiv-II-Varianten im Untersuchungsraum am stärksten entspricht.

Wie in Kapitel 2.1.2 erwähnt, wurden innersprachliche Faktoren in der Studie von Breuer/Wittibschlager (2020: 154) in Bezug auf die Zugehörigkeit der Verben zu Flexionsklassen untersucht. Periphrastische Formen werden von ihnen prozentuell fast doppelt so häufig bei schwachen Verben nachgewiesen wie bei starken und unregelmäßigen Verben. Abbildung 12 zeigt, dass sich eine entsprechende Tendenz zwar für die einfachen Verben, nicht aber für die hier untersuchten Partikelverben belegen lässt. Insgesamt erscheinen Partikelverben mit schwachem Basisverb sogar geringfügig öfter mit synthetischen Varianten. Auch dies lässt darauf schließen, dass Partikelverben, deren Basisverben als einfache Verben den Konjunktiv II ohne Stammmodifikation bilden, leichter in das synthetische Bildungsschema integriert werden können als Partikelverben, deren Basisverben als einfache Verben den Konjunktiv II mit Stammmodifikation bilden (was bei starken Verben vielfach der Fall ist).

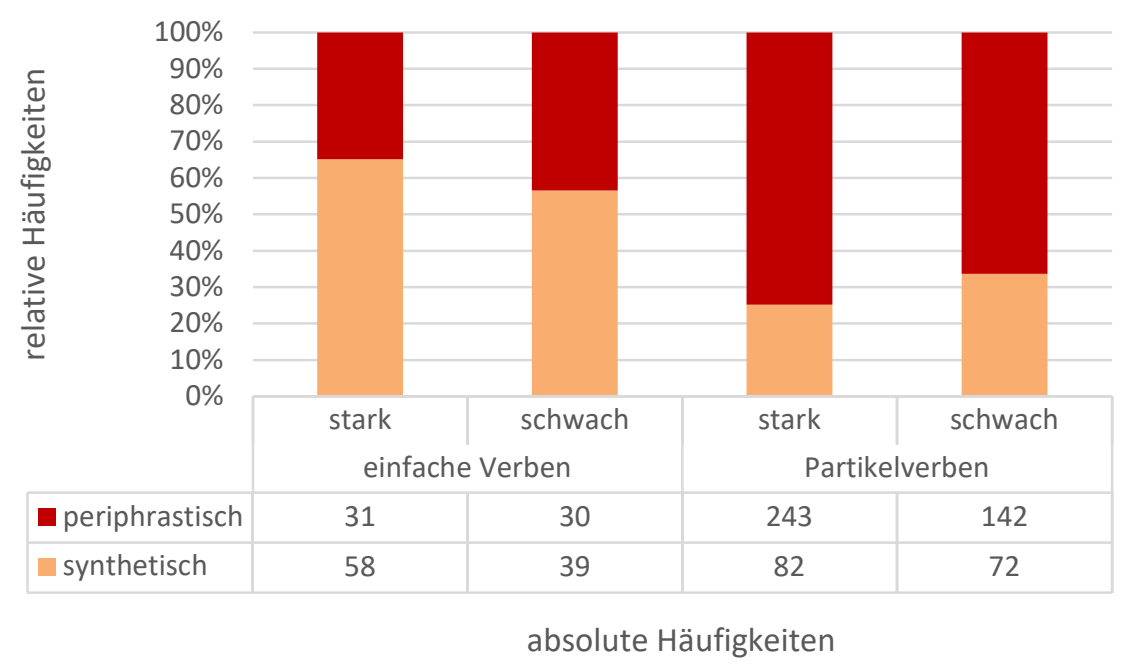

Abbildung 12: Relative (in \%) und absolute Häufigkeiten der untersuchten einfachen Verben $(\mathrm{n}=158)$ und Partikelverben $(n=539)$ in synthetischen und periphrastischen Konstruktionsvarianten im Hinblick auf die Zugehörigkeit zu Flexionsklassen ${ }^{10}$

In Kapitel 4.2 wurde beschrieben, dass es auch auffällige verbspezifische Unterschiede zwischen Partikelverben mit demselben Basisverb gibt. Um diese Unterschiede zu erklären,

\footnotetext{
10 Die Daten zu (-)bringen wurden aufgrund der Zuordnungsschwierigkeit des Verbs zu einer Flexionsklasse ausgenommen (cf. auch Vergeiner/Bülow 2022; Mauser 1998: 353).
} 
müssen syntaktische und semantische Faktoren berücksichtigt werden. Dies wird in weiterer Folge exemplarisch für die Partikelverben aufgeben (trans.), aufgeben (intrans.), (ein)kaufen, annähen, abkaufen, einlesen und angeben diskutiert. Abbildung 13 zeigt, wie häufig bei diesen Verben jeweils synthetische Varianten erscheinen.

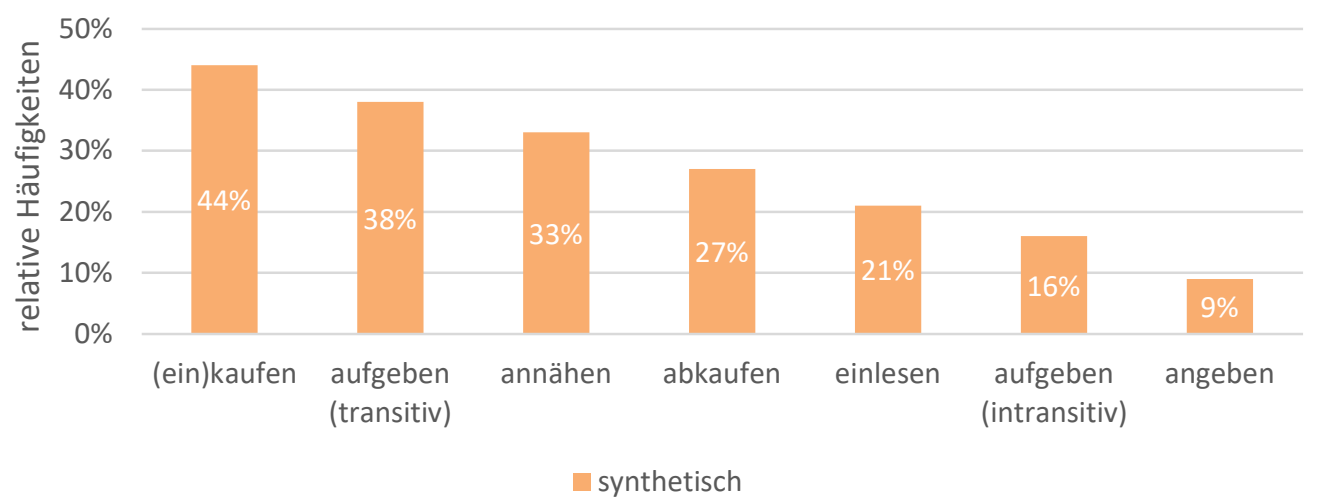

Abbildung 13: Relative Häufigkeiten der Verben (ein)kaufen $(n=15)$, aufgeben (transitiv, $n=5)$, annähen $(n=15)$, abkaufen $(n=13)$, einlesen $(n=9)$, aufgeben (intransitiv, $n=4)$ und angeben $(n=4)$ in synthetischen Konjunktiv-II-Varianten

Synthetische Varianten treten am häufigsten bei einkaufen auf. Erklärbar ist dies durch die Tatsache, dass hier die Linkserweiterung mithilfe der Partikel keinen relevanten Bedeutungsunterschied hervorruft. Dass dem so ist, zeigt sich etwa darin, dass das Verb von den GP der vorliegenden Untersuchung häufiger ohne Partikel realisiert wird (also kaufen statt einkaufen). Auch folgender Beleg mit eingeklammerter Partikel legt nahe, dass die Partikel fakultativ ist:

(1) I kaffat gern mehr Gemüse (ei), oba mia brauchen nid so viel. ,Ich kaufte gerne mehr Gemüse (ein), aber wir brauchen nicht so viel.'(022_AL_jung_w2_1)

Aus der Tatsache, dass die Partikel bei einkaufen weder die grammatischen Eigenschaften (Valenz) noch die Semantik des Partikelverbs stärker modifiziert, ergibt sich eine größere Autonomie von Basisverb und Partikel. Dies dürfte dazu beitragen, dass hier die Unterscheidung zwischen Kontakt- und Distanzstellung weit weniger relevant ist als bei anderen Partikelverben, z. B. abkaufen. Bei abkaufen handelt es sich zwar um ein lexikalisch transparentes Partikelverb (siehe Kapitel 2.2.2), im Gegensatz zu einkaufen kann das Basisverb -kaufen die Bedeutung bei abkaufen aber nicht eigenständig tragen, da die Partikel die Beendigung eines Vorgangs (egressive Wortbildungsbildungsbedeutung) anzeigt (cf. Erben 2006: 87f.; Fleischer/Barz 2012: 382, 398) und infolgedessen beide Bestandteile des Partikelverbs semantisch relevant sind. Die Differenz im Anteil synthetischer Varianten zwischen einkaufen (44\%) und abkaufen (27\%) beträgt immerhin 17 Prozentpunkte.

Bei (-)nähen besteht - anders als bei allen anderen hier analysierten Verben - kein signifikanter Zusammenhang zwischen der morphologischen Bildung (einfaches Verb vs. Partikelverb) und der Verwendung synthetischer oder periphrastischer Varianten. Dies könnte wiederum mit dem geringen Grad an semantischer Modifikation beim Basisverb des Partikelverbs zusammenhängen: Bei Partikelverben, bei denen an- ein Verbinden signalisiert, kennzeichnen häufig auch die Basisverben alleine „eine Annäherung oder Verbindung zweier Größen“ (Fleischer/Barz 2012: 402). Da dies bei nähen der Fall ist, könnte so das ähnliche Variationsverhalten von 
nähen und -nähen plausibilisiert werden. Zu berücksichtigen ist freilich, dass nähen von allen einfachen Verben am häufigsten periphrastisch realisiert wird (cf. auch Vergeiner/Bülow 2022), was den Unterschied zwischen nähen und -nähen zusätzlich verringert.

Das semantisch opake Partikelverb angeben (,prahlen') wurde mit 90,7\% ( $n=39)$ am häufigsten periphrastisch realisiert. Dass bei diesem Verb ein sehr hoher Lexikalisierungsgrad vorliegt, trägt wohl dazu bei, dass hier die Distanzstellung der Wortbestandteile dispräferiert ist. Auch syntaktische Faktoren wie etwa Rektionseigenschaften könnten die Konjunktiv-II-Variation einzelner Partikelverben steuern. Darauf deuten die Befunde zum Verb aufgeben hin, das je einmal als transitives (etw. aufgeben) und einmal als intransitives Partikelverb erhoben wurde: Im Vergleich zur transitiven Variante wurde aufgeben als intransitives Verb $(\mathrm{n}=21$; entspricht 84\%) um 22 Prozentpunkte häufiger periphrastisch realisiert. Dass die transitive Form eher synthetisch realisiert wird, kann dadurch erklärt werden, dass es die Akkusativrektion des zugrundeliegenden Verbs geben beibehält. Damit dürfte bei transitivem aufgeben leichter auf zugrundeliegendes geben und dessen synthetische Konjunktiv-II-Form gabat zurückgegriffen werden können. Auch semantisch ist transitives aufgeben deutlich transparenter und zugleich weniger stark lexikalisiert als intransitives aufgeben.

In anderen Fällen kann die Präferenz für eine bestimmte Konjunktiv-II-Variante nicht (ausschließlich) auf Faktoren wie Lexikalisierung bzw. semantische (In-)Transparenz rückgeführt werden. Stattdessen müssen weitere sozio-pragmatische Faktoren berücksichtigt werden. Dass lexikalisch transparente Partikelverben nicht zwangsläufig häufiger synthetisch realisiert werden als opake, bezeugen bspw. die transparenten Partikelverben ablesen und einlesen, die nach angeben am seltensten synthetisch realisiert wurden. Die Präferenz für periphrastische Formen könnte mit den außersprachlichen Verwendungsbereichen zusammenhängen, mit denen diese Verben verbunden sind: So wurde etwa einlesen in der Phrase sich in ein Thema einlesen erhoben, welche einem literalen Verwendungsbereich zuzuordnen ist. Periphrastische Formen stellen, im Vergleich zu synthetischen, die regiolektaleren und damit auch standardnäheren Varianten dar (cf. Vergeiner/Bülow 2022), weshalb sich Verben wie einlesen und ablesen, die mit standardsprachlichen Verwendungsbereichen assoziiert sind, gut in das periphrastische Bildungsschema einfügen.

In weiterer Folge sollen noch die sprachgeografischen und soziolinguistischen Steuerungsfaktoren diskutiert werden. In Bezug auf sprachgeografische Faktoren ist zunächst zu betonen, dass in jedem einzelnen Erhebungsort periphrastische Formen häufiger bei Partikelverben auftreten als bei einfachen Verben, was unterstreicht, dass es sich um ein robustes Ergebnis handelt. Da unter den untersuchten einfachen Verben mit der Ausnahme von St. Michael (Lungau) in allen Untersuchungsorten synthetische Varianten überwiegen, kann die von Vergeiner/Bülow (2022) dokumentierte starke Verankerung der synthetischen Bildungsstrategie im Bundesland Salzburg bestätigt werden.

Zwar gibt es Unterschiede zwischen den einzelnen Orten, diese lassen sich aber kaum auf die Dialektgrenzen zwischen dem Süd-, Südmittel- und Mittelbairischen (nach Wiesinger 1983) zurückführen (cf. für ein ähnliches Ergebnis Vergeiner/Bülow 2022). Einzelne Unterschiede zwischen den Untersuchungsorten können eher mit soziodemographischen Faktoren erklärt werden: So fällt auf, dass nur im Pinzgauer Untersuchungsort Embach bei den Partikelverben 
mit 51,6\% $(n=48)$ synthetische Varianten gegenüber periphrastischen dominieren. Mit weniger als 610 Einwohner*innen ist das Bergdorf Embach der einwohnerschwächste Untersuchungsort dieser Studie, der zusätzlich besonders verkehrsabgeschieden ist. Dass hier sogar unter den Partikelverben synthetische Formen knapp vor periphrastischen überwiegen, dürfte dem sprachlichem Konservativismus des Ortes geschuldet sein. In St. Michael im Lungau wurden sowohl bei den einfachen Verben als auch bei den Partikelverben von allen Untersuchungsorten am häufigsten periphrastische Formen dokumentiert. Eine sehr gute Verkehrsanbindung (A10 Tauernautobahn, B96, B99) könnte ein Grund für die starke Präferenz der innovativen periphrastischen Formen sein. Zudem ist St. Michael, nach Einwohner*innen gemessen, die zweitgrößte Gemeinde im Lungau.

Eine solche Interpretation der sprachgeografischen Unterschiede steht in Einklang mit den Befunden zu den soziolinguistischen Steuerungsfaktoren. Diese wurden im Rahmen dieser Studie im Hinblick auf die Variablen Alter und Geschlecht untersucht.

Sowohl bei den einfachen Verben als auch bei den Partikelverben ergibt sich ein statistisch signifikanter Alterseffekt: Jüngere Personen neigen stärker zu periphrastischen Konstruktionsvarianten als ältere. Interessanterweise nehmen dabei sowohl würde-Periphrasen ohne -at-Suffix (wie bei Breuer/Wittibschlager 2020) als auch die einfache tun-Periphrase (wie bei Vergeiner/Bülow 2022) bei jüngeren GP stark zu. Obwohl die unsuffigierte würde-Periphrase von jungen GP etwa dreimal so häufig gebraucht wird wie von älteren, stellt die Variante mit tunAuxiliar ohne -at-Suffix die eindeutig präferierte periphrastische Form dar (siehe Kapitel 4.4).

Das Geschlecht der Gewährspersonen erweist sich indes weder bei den untersuchten einfachen Verben noch bei den Partikelverben als statistisch signifikanter Einflussfaktor. Dass Frauen innovativere Varianten präferieren und damit als Trägerinnen des Sprachwandels fungieren (cf. Chambers/Trudgill 1998), kann somit in dieser Studie, wie auch bei Vergeiner/Bülow (2022), nicht bestätigt werden.

\section{$6 \quad$ Fazit}

Ziel des vorliegenden Beitrags war es, mithilfe eines Fragebogens Variation und Wandel der Konjunktiv-II-Bildung in den Dialekten Salzburgs zu untersuchen. Da bisher nur wenige Erkenntnisse über den Einfluss (wortbildungs-)morphologischer und syntaktischer Faktoren vorliegen, wurde in dieser explorativen apparent-time-Studie die Konjunktiv-II-Bildung von Partikelverben fokussiert. Deutlich wurde, dass sich diese signifikant von derjenigen bei einfachen Verben unterscheidet: Die 15 abgefragten Partikelverben wurden von den GP deutlich häufiger mit periphrastischen Varianten gebildet als die entsprechenden einfachen Verben.

Diese Tendenz hängt zumindest teilweise mit den semantisch-lexikalischen und morpho-syntaktischen Eigenschaften von Partikelverben zusammen. So scheinen periphrastische Varianten bei Partikelverben u. a. deshalb bevorzugt zu werden, weil die periphrastische Bildungsstrategie (mit den Auxiliaren täte und würde) im Gegensatz zur synthetischen eine Kontaktstellung von Verbpartikel und Basisverb erlaubt. Damit bestätigt sich für die Konjunktiv-II-Bildung bei Partikelverben Behaghels (1932: 4) Annahme, dass Sprecher*innen dazu tendieren, „geistig eng Zusammengehöriges“ (hier: Verbpartikel und Basisverb) adjazent im Satz zu positionieren. 
Trotz dieser allgemeinen Tendenz zeigen sich in dieser Studie Unterschiede zwischen einzelnen Partikelverben: So besteht ein starker Zusammenhang zwischen der Konjunktiv-II-Bildung einfacher Verben (z. B. bringen) und der Konjunktiv-II-Bildung bei denselben Verben als Basisverben bei Partikelverbbildungen (z. B. (-)bringen bei abbringen oder einbringen). Wenn z. B. bei einfachen Verben starke synthetische Bildungen mit Stammmodifikation dominieren (z. B. bei bringen $>$ bracht), besteht eine stärkere Tendenz zur periphrastischen Bildungsstrategie bei den entsprechenden Partikelverben, als wenn die zugrundeliegenden einfachen Verben schwache Konjunktiv-II-Bildungen aufweisen (z. B. bei sagen > sagat). Auch der Lexikalisierungsgrad der Partikelverben spielt eine wichtige Rolle, wobei ein hoher Lexikalisierungsgrad bei Partikelverben mit einer verstärkten Präferenz für die morpho-syntaktische Kontaktstellung zwischen Verbpartikel und Basisverb einhergeht. Umgekehrt scheint eine hohe Transparenz und/oder ein geringerer Einfluss der Verbpartikel auf die Gesamtbedeutung des Partikelverbs mehr Möglichkeiten zur Distanzstellung zu eröffnen. Des Weiteren wurde in dieser Untersuchung herausgearbeitet, dass auch die Valenzeigenschaften der Partikelverben einen Einfluss auf die Konjunktiv-II-Variation nehmen können.

Hinsichtlich der sprachgeografischen Verteilung zeigen sich zwar Unterschiede zwischen den einzelnen Orten, diese korrelieren aber nicht mit den traditionellen Dialektgrenzen. Sie lassen sich eher auf die soziodemographischen Eigenheiten (Einwohnerzahl, Verkehrsanbindung, Tourismus, etc.) der Untersuchungsorte zurückführen.

In Übereinstimmung mit anderen Untersuchungen zur Konjunktiv-II-Bildung in den bairischen Dialekten Österreichs (cf. Breuer/Wittibschlager 2020; Vergeiner/Bülow 2022) erweist sich auch in dieser Studie das Alter der GP als relevanter Faktor: Sowohl bei einfachen Verben als auch bei Partikelverben ergibt sich ein statistisch signifikanter Unterschied, wobei die periphrastischen Varianten stärker von den jüngeren GP präferiert werden. Im Zuge dessen nimmt vor allem die Verwendung des würde-Auxiliars (ohne -at-Suffix) zur Konjunktiv-II-Bildung stark zu. Nichtsdestoweniger überwiegen bei den periphrastischen Varianten jüngerer GP nach wie vor Bildungen mit dem täte-Auxiliar ohne -at-Suffix.

Diese Studie konnte zeigen, dass neben dem Faktor Alter, der auf aktuellen Sprachwandel hindeutet, insbesondere innersprachliche Faktoren die Konjunktiv-II-Bildung in den bairischen Dialekten Salzburgs beeinflussen.

\section{Literaturverzeichnis}

Behaghel, Otto (1932): Deutsche Syntax: eine geschichtliche Darstellung. Band IV. Wortstellung-Periodenbau. Heidelberg: Carl Winter.

Breuer, Ludwig Maximilian/Wittibschlager, Anja (2020): „The variation of the subjunctive II in Austria. Evidence from urban and rural analyses”. Linguistic Variation 20/1: 136-171.

Burger, Harald (2003): Phraseologie. Eine Einführung am Beispiel des Deutschen. 2., überarbeitete Auflage. Berlin: Erich Schmidt Verlag. (= Grundlagen der Germanistik 36).

Chambers, Jack K./Trudgill, Peter (1998): Dialectology. Second Edition. Cambridge: Cambridge University Press.

DiÖ = Spezialforschungsbereich (FWF F60) ,Deutsch in Österreich. Variation - Kontakt Perzeption". dioe.at [09.02.2022]. 
Dürscheid, Christa/Sutter, Patrizia (2014): „Grammatische Helvetismen im Wörterbuch“. Zeitschrift für angewandte Linguistik 60/1: 37-65.

Eisenberg, Peter (2006): Das Wort. Grundriss der Deutschen Grammatik. Stuttgart: Metzler.

Erben, Johannes (2006): Einführung in die deutsche Wortbildungslehre. 5., durchgesehene und ergänzte Auflage. Berlin: Erich Schmidt Verlag. (= Grundlagen der Germanistik 17).

Felfe, Marc (2012): Das System der Partikelverben mit „, an “. Eine konstruktionsgrammatische Untersuchung. Berlin/Boston: de Gruyter. (=Sprache und Wissen 12).

Fingerhuth, Matthias (2019): „Grammatische Variation im deutschen und schweizerischen Standarddeutsch des 20. Jahrhunderts“. In: Bülow, Lars/Fischer, Ann Kathrin/Herbert, Kristina (eds.): Dimensions of Linguistic Space: Variation - Multilingualism - Conceptualisations. Dimensionen des sprachlichen Raums: Variation-Mehrsprachigkeit-Konzeptualisierung. Berlin, Lang: 105-125. (= Schriften zur deutschen Sprache in Österreich 45).

Fleischer, Jürg/Kasper, Simon/Lenz, Alexandra N. (2012): „Die Erhebung syntaktischer Phänomene durch die indirekte Methode: Ergebnisse und Erfahrungen aus dem Forschungsprojekt „Syntax hessischer Dialekte“ (SyHD)“. Zeitschrift für Dialektologie und Linguistik 79/1: 2-42.

Fleischer, Wolfgang/Barz, Irmhild (2012): Wortbildung der deutschen Gegenwartssprache. 4. Auflage; völlig neu bearbeitet von Irmhild Barz unter Mitarbeit von Marianne Schröder. Berlin/Boston: de Grutyer.

Glauninger, Manfred (2008): „Synthetische und analytische ,Konjunktiv 2'-Formen im Wiener Nonstandard-Deutsch“. In: Patocka, Franz/Seiler, Guido (eds.): Dialektale Morphologie, dialektale Syntax. Beiträge zum 2. Kongress der Internationalen Gesellschaft für Dialektologie des Deutschen (Wien, 20.-23. September 2006). Wien, Praesens: 233-247.

Glauninger, Manfred (2010): „Zur ,(Morpho-)Pragmatik“ von „Konjunktiv 2“-Formen im Wiener Nonstandard-Deutsch“. In: Pohl, Heinz Dieter (ed.): Akten der 10. Arbeitstagung für bayerisch-österreichische Dialektologie (Klagenfurt, September 2007). Wien, Praesens: 98 107.

Glauninger, Manfred (2011): „Zum honorativen Konjunktiv 2 als Modalisierungsoption. Aspekte einer funktionalen Typologie des Wiener Deutsch“. In: Christen, Helen/Patocka, Franz/Ziegler, Evelyn (eds.): Struktur, Gebrauch und Wahrnehmung von Dialekt. Beiträge zum 3. Kongress der Internationalen Gesellschaft für Dialektologie des Deutschen (IGDD) (Zürich, 7.-9. September 2009). Wien, Praesens: 47-57.

Küng, Katharina (1972): Die festen Verbalpräfixe in den tirolischen Mundarten. Bestand-Verteilung - Funktion. Dissertation, Universität Innsbruck.

Lenzhofer, Melanie (2017): Jugendkommunikation und Dialekt. Syntax gesprochener Sprache bei Jugendlichen in Osttirol. Berlin/Boston: de Gruyter. (=Empirische Linguistik/Empirical Linguistics 6).

Mauser, Peter (1998): Die Morphologie im Dialekt des Salzburger Lungaus. Frankfurt am Main: Lang. (= Schriften zur deutschen Sprache in Österreich 27).

Niehaus, Konstantin (2015): „Areale Variation in der Syntax des Standarddeutschen. Ergebnisse zum Sprachgebrauch und zur Frage Plurizentrik vs. Pluriarealität“. Zeitschrift für Dialektologie und Linguistik 82/2: 133-168. 
SADS = Glaser, Elvira (ed.) (2021): Syntaktischer Atlas der deutschen Schweiz (SADS). Band 1: Einleitung und Kommentare. Bearbeitet von Elvira Glaser et al. Band 2: Karten. Bearbeitet von Sandro Bachmann et al. Tübingen: Narr.

Schabus, Sieglinde (1982): Die Präfixverben in den südbairischen Dialekten Kärntens. Eine Untersuchung zur Wortbildung. Wien: Braumüller. (= Schriften zur deutschen Sprache in Österreich 8).

Stiebels, Barbara (1996): Lexikalische Argumente und Adjunkte. Zum semantischen Beitrag von verbalen Präfixen und Partikeln. Berlin: Akademie Verlag. (= studia grammatica 39).

Stöckle, Philipp (2020): „Dialektvariation an der Schnittstelle von Syntax, Morphologie und Lexik - Der Konjunktiv II in den bairischen Dialekten Österreichs und Südtirols“. In: Christen, Helen et al. (eds.): Regiolekt - Der neue Dialekt? Stuttgart, Steiner: 149-173. (= Zeitschrift für Dialektologie und Linguistik, Beihefte 182).

SyHD = Fleischer, Jürg/Lenz, Alexandra N./Weiß, Helmut (2017): SyHD-atlas. Konzipiert von Ludwig M. Breuer. Unter Mitarbeit von Katrin Kuhmichel et al. Marburg/Wien/Frankfurt am Main. dx.doi.org/10.17192/es2017.0003.

Variantengrammatik des Standarddeutschen (2018). Ein Online-Nachschlagewerk. Verfasst von einem Autorenteam unter der Leitung von Christa Dürscheid, Stephan Elspaß und Arne Ziegler. mediawiki.ids-mannheim.de/VarGra/index.php [22.07.2021].

Vergeiner, Philip C./Bülow, Lars (2022): „Der Konjunktiv II in den ruralen Basisdialekten Österreichs. Quantitative und qualitative Befunde“. Linguistik Online: in diesem Band.

WBÖ = Institut für Corpuslinguistik und Texttechnologie (ICLTT) (vormals Institut für Österreichische Dialekt- und Namenlexika) (ed.) (1970-): Wörterbuch der bairischen Mundarten in Österreich. Wien: Verlag der Österreichischen Akademie der Wissenschaften (= Bayerisch-österreichisches Wörterbuch: I. Österreich).

Wiesinger, Peter (1983): „Die Einteilung der deutschen Dialekte“. In: Besch, Werner et al. (eds.): Dialektologie. Ein Handbuch zur deutschen und allgemeinen Dialektforschung. Zweiter Halbband. Berlin/New York, de Gruyter: 807-900. (= Handbücher zur Sprach- und Kommunikationswissenschaft 1.2). 